\title{
An empirical study on the regulated Chinese agricultural commodity futures market based on skew Ornstein-Uhlenbeck model
}

December 16, 2020

\begin{abstract}
This paper describes the regulated agricultural commodity futures market of China, focusing on six actively traded futures: corn, strong gluten wheat, No. 1 soybean, soymeal, cotton, and white sugar. A novel skew Ornstein-Uhlenbeck model is employed to characterize price dynamics with government controls. The empirical analysis reveals significant skew phenomena in these six futures and indicates that the price dynamics are influenced by state policy. The observed skew phenomena are most notable in grain futures, with relatively weaker, but statistically significant, evidence of skew phenomena in oilseed and soft futures markets. In addition, generalized quasilikelihood ratio tests show that the skew Ornstein-Uhlenbeck model is superior to the OrnsteinUhlenbeck model.
\end{abstract}

Keywords: Agricultural commodity futures, Skew Ornstein-Uhlenbeck processes

JEL Codes: G13, G18, Q14 


\section{Introduction}

Because of its strong and consistent demand, China has become the world's largest importer and consumer of commodities. Considering the important role China plays in international commodity markets and the increasing risk management demand from entity enterprises, Chinese commodity futures market has witnessed tremendous growth in recent years. Among the global commodity futures and options exchanges, the trading volume of the Shanghai Futures Exchange (SHFE) ranked first, while the Dalian Commodity Exchange (DCE) ranked second, and the Zhengzhou Commodity Exchange (CZCE) ranked fourth in $2019^{1}$. Prices on Chinese exchanges are becoming increasingly influential. This paper focuses on agricultural commodity futures in particular. Chinese agricultural futures accounted for $80 \%$ of the world's top five contracts in $2019^{1}$. However, to our knowledge, they have not been thoroughly investigated. The modeling of agricultural derivatives under Chinese heterogeneous characteristics is still undeveloped. This paper chooses six Chinese agricultural commodity futures that represent the most liquid contracts and are important agricultural commodities, in part because they have become an important part of overall global futures markets, with tremendous trading volume.

However, compared to foreign commodities markets, regulation is very common in Chinese markets. For agricultural commodities in particular, policies such as temporary reserve and minimum purchase policies apply. Government authorities seek to implement macro-interventions on the prices of agricultural commodities, with price caps and/or price supports. Such policies place invisible supports under the market. Under such policies, when the price of a commodity declines to a certain level, it will then show a high probability of an increasing trend. Therefore, the price dynamics are influenced by government policy. Traditional models assume that price dynamics of assets move upwards with the same probability as downwards, and that such phenomena cannot be captured. Accordingly, we need different models to characterize Chinese agricultural commodity futures. This observation in part motivates our specific setting of the skew Ornstein-Uhlenbeck (skew OU) model, first proposed by Wang et al. (2015). Similar to skew Brownian motion (SBM) introduced by Itô and Mckean (1965), the skew OU model adds a local time term to the Ornstein-Uhlenbeck (OU) model to capture both mean-reverting and regulatory characteristics of dynamics. The local time term has two parameters, skew level and skew probability. When the process hits the skew level, it moves up with skew probability and moves down with 1 minus skew probability. In general, skew probability tends to differ from 0.5, reflecting specific controlled and regulated characteristics. We define the phenomenon in which dynamics move upwards with different probability to moving downwards when hitting a certain level as "skew phenomenon". Specifically, the skew OU model will reduce to the OU model if skew probability equals 0.5 , and to the reflected $\mathrm{OU}$ model if skew probability equals 0 or 1 .

The mean-reverting feature of commodity prices is pointed out in a number of studies. Bessembinder et al. (1995) shows that for agricultural commodities, the observed mean reversion is large in magnitude compared to other commodities and arises from positive comovement between prices and implied cash flow yields. Schwartz (1997) gives prices of commodity and futures contracts when taking into account mean reversion. This author suggests that it is quite important to consider mean reversion in prices when implementing capital budgeting. If mean reversion is neglected, we would induce investment too late. Schwartz and Smith (2000) develops a model of commodity prices that allows

\footnotetext{
${ }^{1}$ Data are from the Futures Industry Association (FIA).
} 
for mean reversion in short-term prices while simultaneously allowing uncertainty in the equilibrium level to which prices revert. Geman (2005), in an introduction to commodity derivatives markets and modeling, points out that mean reversion is a salient feature of commodity prices. Casassus and Collin-Dufresne (2005) identify two sources of mean reversion for commodity prices. Li and Linetsky (2014) holds that the geometric OU model plays the same role in commodity markets as the geometric Brownian motion model plays in the equity markets.

However, as stated above, in Chinese agricultural markets, regulation is common. The OU model has no way to characterize this regulatory phenomenon, while the skew OU model can capture specific regulated characteristics and preserve mean-reverting features at the same time. A large body of literature examines regulated markets. Motivated by the European Monetary System (EMS) exchange rate under its target zone, Krugman (1991) first modeled the target zone of foreign exchange rates, which is perfectly credible. Also, Bo et al. (2013, 2011a,b), Bo (2013) , Lee and Song (2016), Yang et al. (2016), Han et al. (2016, 2019) and Cai and Yang $(2018,2020)$ examine reflected diffusion processes that can capture perfectly credible boundaries. However, a process may breach the officially declared boundaries of the target zone. There may be a "soft" floor or ceiling. Svensson (1991) and Farnsworth and Bass (2003) study semi-credible interest rates targets. Avriel et al. (2013) models partially credible inflation target regimes. Similar phenomena can be observed in Chinese agricultural commodity markets. Although the minimum purchase policy is present, there may be hysteresis from the intervention. For soft target zones, skew models can capture a controlled probability of the process wandering beyond particular boundaries. Wang et al. (2015) assumes the logarithmic price of an asset follows the skew OU process. The price of a defaultable zero coupon bond with zero recovery and conditional default probability under incomplete information is calculated. Zhuo and Menoukeupamen (2017) assume that the short rate is given by a generalized skew OU model with discontinuous drift coefficient and provide corresponding bond prices and European/American bond option prices. In terms of empirical research based on the skew OU model, Zhuo (2018) applies the skew OU model to the Chinese short-term interest rate market and finds that skew levels act as invisible pressure levels that are significant and persistent. Bai and Guo (2019) find that skew probability is quite different from 0.5 for stock index markets. In addition, some literature examines the impact of policy on financial markets. Mohanty and Mishra (2020) study the effect of regulatory reform on Indian agricultural commodity futures market. Klomp (2020) investigate policy impacts on the return of agricultural commodity futures in the EU. Perera et al. (2020) study a specific agricultural commodity, tea, and analyze the corresponding regulatory environment. Chen and Chiang (2020) investigate policy uncertainty in China's stock market. Jian et al. (2018) and Huo and Ahmed (2018) research the stock index futures market in the context of China.

Several works implement the skew OU model in the pricing of derivatives and capture skew phenomena in interest markets, but not for commodity markets. All the works cited above indicate the validity of the skew OU model. Our aim here is to study skew phenomena in agricultural commodity futures market, and to show whether the skew OU model is superior to the OU model. Thus, we assume that the logarithm of agricultural commodity futures prices follow the skew OU model and fits historical daily settlement data for corn, strong gluten wheat, No. 1 soybean, soymeal, white sugar, and cotton futures. We design a significance test to investigate whether skew probabilities in these markets are significantly different from 0.5. Further, we conduct a generalized quasi-likelihood ratio test to determine whether the skew OU model is more suitable than the OU model. Our principal 
findings indicate that there are indeed significant skew phenomena in Chinese agricultural commodity futures market. Understanding skew phenomena in agricultural commodity futures market is important for two main reasons. First, it allows us to capture specific regulation-related characteristics in these markets. Second, it indicates the effectiveness of government regulatory policy, as reflected by the magnitude of skew probabilities. The remainder of this paper proceeds as follows. Section 2 describes our data and sets forth our motivation for choosing the skew OU model. Section 3 provides a brief introduction to our models. Section 4 discusses our empirical results. Finally, Section 5 summarizes our results.

\section{Data, Summary Statistics, and Characteristics of Price Changes}

\subsection{Data}

This paper focuses on the six agricultural commodities that have the most actively traded futures studied in the literature. They can be separated into three groups: corn and strong gluten wheat are grain products, No. 1 soybean and soymeal are oilseed products, and white sugar and cotton represent soft products ${ }^{2}$. Among grain products, China is the world's second-largest corn producer, second only to the U.S. ${ }^{3}$, and Chinas trading volume in corn futures ranked fifth in $2019^{4}$. Wheat is the secondlargest grain crop in the world, second only to corn. Wheat futures are divided into strong gluten, medium gluten, and weak gluten. Trading volumes of strong gluten wheat futures are much higher than common wheat futures. Among oilseed products, China is the world's largest importer and consumer of soybeans, accounting for about $60 \%$ of global soybean trade in $2019^{5}$. The underlying product of the No. 1 soybean contract is non-transgenic soybean. The No. 1 soybean contract is chosen because it represents domestic non-transgenic soybeans, while No. 2 soybean contracts represent soybean for oil extraction mainly from imported transgenic soybeans. The delivery standards for imported transgenic soybeans are relatively strict. Therefore, trading volumes and open positions for No. 1 soybeans are much larger. Market liquidity of futures contracts affects the quality of futures data. As a result, the No. 1 soybean contract is more suitable for research. Soymeal is a by-product of soybean oil extraction. The oil yield of transgenic soybean is much higher than that of non-transgenic soybean, and without doubt, transgenic soybean is used mainly for oil extraction. Therefore, soymeal prices are highly correlated with transgenic soybean. The DCE's soymeal contract remained the world's largest agricultural contract by volume for eight consecutive years since $2010^{4}$. Among soft products, China ranks first in production, input, and consumption of cotton in the world ${ }^{6}$. The CZCE sugar contract ranked fourth in global agricultural derivatives markets in $2019^{4}$. We are interested in these six particular agricultural markets in part because of their growing importance in the world market.

Our data on agricultural commodity futures are obtained from the Wind database, which contains daily futures settlement prices. Data are available for different sample periods depending on the launch date of contracts. The sample periods all end on December 22, 2018. As futures contracts expire after

\footnotetext{
${ }^{2}$ Our classification is similar to that of the Institute for Financial Markets.

${ }^{3}$ Information is from DCE.

${ }^{4}$ Data are from FIA .

${ }^{5}$ Data are from United States Department of Agriculture (USDA).

${ }^{6}$ Information is from CZCE.
} 
several months, we construct a continuous sequence by choosing the data from the dominant future contracts with the largest trading volume. We choose futures data instead of spot data for two reasons. First, futures data are undoubtedly of higher quality, because they represent real trading prices with fewer missing values. Second, futures data are more uniform than spot prices. Table 1 lists the six agricultural commodities futures in our analysis, together with contract names, the exchanges on which they trade, transaction codes, and the starting date of available daily settlement data.

Table 1: Information for six agricultural commodity futures

\begin{tabular}{|c|c|c|c|}
\hline Commodity & Exchange traded & Transaction code & Launch date \\
\hline \multicolumn{4}{|l|}{ Grains } \\
\hline Corn & DCE & $\mathrm{C}$ & 2004.09 .22 \\
\hline Strong gluten wheat & $\mathrm{CZCE}$ & $\mathrm{H}$ & 2003.03 .28 \\
\hline \multicolumn{4}{|l|}{ Oilseeds } \\
\hline No. 1 Soybean & DCE & $\mathrm{A}$ & 2002.03 .15 \\
\hline Soymeal & DCE & M & 2000.07 .17 \\
\hline \multicolumn{4}{|l|}{ Softs } \\
\hline Cotton & $\mathrm{CZCE}$ & $\mathrm{CF}$ & 2004.06 .01 \\
\hline White sugar & $\mathrm{CZCE}$ & SR & 2006.01 .16 \\
\hline
\end{tabular}

Notes: This table presents the six commodity futures employed in this study. The six futures are classified into three categories and traded on two main commodity exchanges in China. Launch date is the beginning of the sample period. Source: Wind database.

\subsection{Summary Statistics}

Table 2 reports mean, median, minimum, maximum, standard deviation, skewness, and kurtosis values as well as the Jarque-Bera test results of the logarithm of six futures prices. The mean values of logarithmic prices range from 7.5188 to 9.6583 , while volatility varies from 0.1916 to 0.2229 . The results show that futures have relatively higher returns but are more risky. The skewness of the six futures logarithmic prices differs from 0 and kurtosis is greater than 3. Jarque-Bera tests reject the null hypothesis that their distributions are normal at the $1 \%$ significance level. The skew OU model describes the non-normal characteristics of assets to some extent.

Table 2: Descriptive statistics for six agricultural commodity futures

\begin{tabular}{lcccccc}
\hline & Corn & Strong gluten wheat & No. 1 soybean & Soymeal & Cotton & White sugar \\
\hline Mean & 7.5188 & 7.7308 & 8.1920 & 7.8950 & 9.6583 & 8.5297 \\
Median & 7.4997 & 7.8178 & 8.2372 & 7.9359 & 9.6150 & 8.5529 \\
Max. & 7.8493 & 8.0690 & 8.5574 & 8.3770 & 10.4450 & 8.9286 \\
Min. & 7.0317 & 7.2984 & 7.6039 & 7.3499 & 9.2098 & 7.9417 \\
Std. Dev. & 0.2154 & 0.1916 & 0.2229 & 0.2196 & 0.2137 & 0.2169 \\
Skewness & -0.2347 & -0.4135 & -0.6953 & -0.6178 & 1.0019 & -0.3848 \\
Kurtosis & 2.1113 & 1.8247 & 2.5326 & 2.7495 & 4.1241 & 2.4297 \\
J-B & 145.7072 & 328.8878 & 365.6029 & 296.4432 & 779.4788 & 120.4420 \\
Prob. & 0.0000 & 0.0000 & 0.0000 & 0.0000 & 0.0000 & 0.0000 \\
Observation & 3462 & 3822 & 4077 & 4476 & 3544 & 3151 \\
\hline
\end{tabular}

Notes: This table reports descriptive statistics for the six commodity futures used in this study. The sample spans from the launch date of each contract to December 22, 2018. Source: Wind database. 


\subsection{Characteristics of Price Changes}

Figures 1-6 below plot the logarithm of agricultural commodity futures prices together with their international counterparts. The Chicago Board of Trade (CBOT) is the world's largest futures market for agricultural commodities and the center for pricing global agricultural commodities. The CBOT futures price has become the benchmark price for the global corn, wheat, soybean, and soymeal trade. Therefore, daily settlement prices for international corn, wheat, soybean, and soymeal futures are obtained from the CBOT. The Intercontinental Exchange (ICE) is the recognized cotton and sugar pricing center. Daily settlement prices of international cotton and sugar contracts are obtained from ICE. We perform two rounds of data processing to obtain our paired data. The first is trading-day processing. Due to the inconsistency of holidays in different markets and the absence of trading on certain days, to maintain data pairing, all non-paired data are deleted. The second is the handling of quote units. The quotation units in the Chinese futures market are all in RMB/ton. To maintain consistency, futures quotations from corresponding international markets are uniformly converted into $\mathrm{RMB} /$ ton through the exchange rate of RMB/USD on the same day, along with the corresponding conversion relationship of weight units.

Figure 1, indicates that it took only about 2 years for the logarithm of corn futures prices to rise from 7.3 in the beginning of 2009 to 7.8 at the end of 2010. As a result of the temporary reserve policy, domestic corn prices showed a steady upward trend. Then, prices of corn futures experienced relatively small shocks for about three years. Corn prices in China are relatively independent from international prices because of a strict import quota ${ }^{7}$. Since 2012, international corn prices have peaked and entered a long bear market, while domestic corn prices stayed at a relatively stable level. The main reason for this is powerful government intervention. However, from February 2015 to August 2015, domestic corn prices dropped dramatically. The temporary reserve policy began to enter a new stage of reform, and the government pushed to cut stocks. After 2016, corn prices began a new round of increase following the reform. Strong gluten wheat futures, shown in Figure 2, show price fluctuations, but the long-term upward trend is very clear compared to its international counterpart. The market price of wheat, on the whole, is centered on the minimum purchase price set by the government. As planting costs rose year by year, to protect the interests of farmers, China gradually raised the minimum purchase price. At the same time, the country strictly limited import volume ${ }^{7}$ and thus the price was not very market-based. Domestic wheat prices stood in stark contrast to the sharp fluctuations of international wheat prices shown in Figure 2.

Figure 3 shows two drastic fluctuations in the dynamics of No. 1 soybean futures. The first one occurred from August 2003 to August 2004, known as the soybean crisis. The USDA announced that soybean stocks were at a 20-year low in August 2003. In response, the price of soybeans surged to a 30-year high in April 2004. Chinese companies began panic purchases of soybeans at relatively high prices. However, after completing these purchases, soybean prices fell sharply in just two months, causing soybean enterprises in China to suffer huge losses. In addition, the financial crisis of 2008 gave rise to another drastic drop in soybean prices. The Chinese government implemented a temporary reserve policy for soybeans from 2008 to 2013, which formed strong support for soybeans. During this period, dynamic fluctuations in the Chinese soybean futures market were smaller compared to international counterparts. The prices of soymeal shown in Figure 4 also experienced two similar

\footnotetext{
${ }^{7}$ According to the Ministry of Agriculture, domestic policies for principal food commodities (corn, wheat, and rice) set the self-sufficiency rate at about $95 \%$.
} 


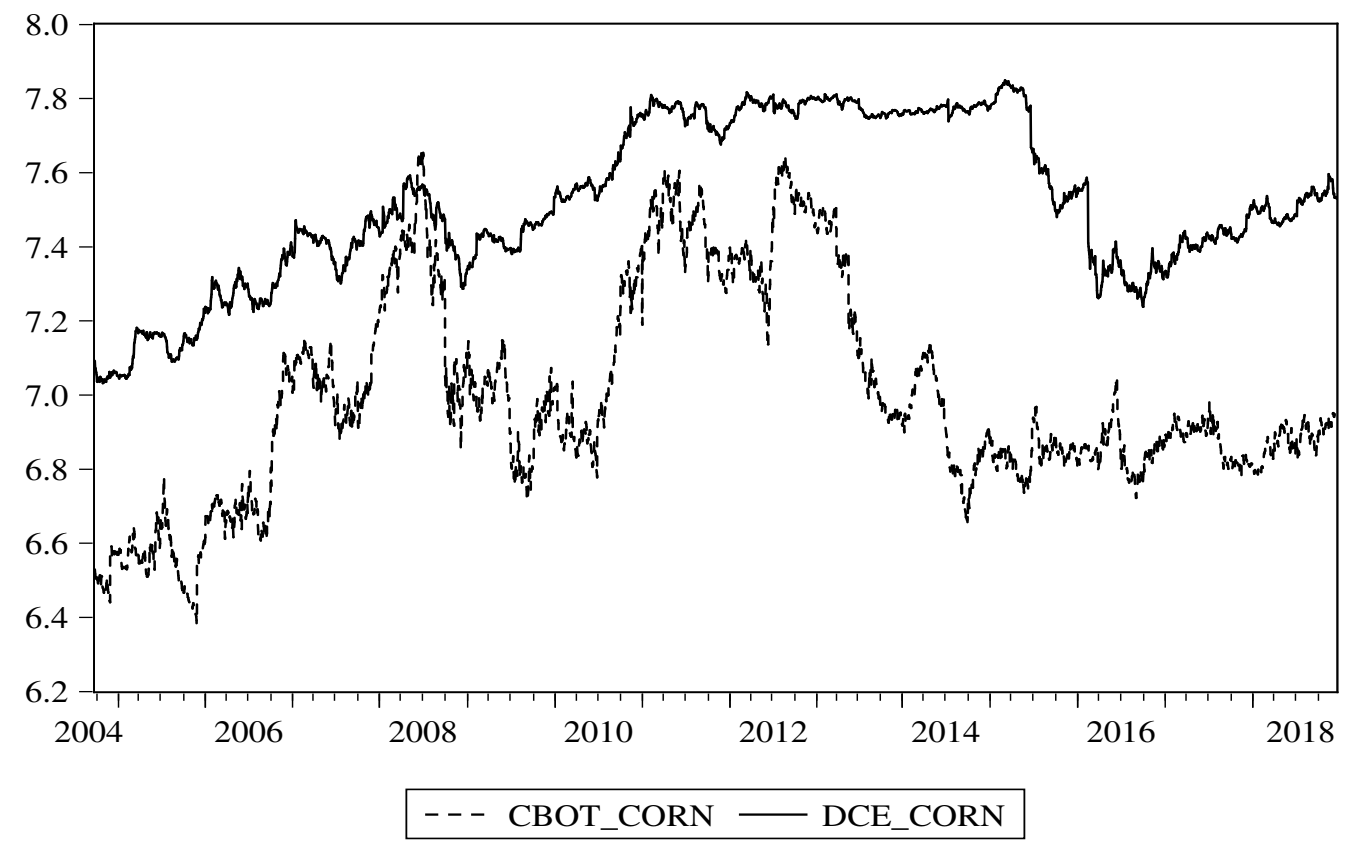

Figure 1: Logarithm of corn futures prices

Notes: This figure plots logarithmic prices of corn futures in DCE (solid curve) together with their international counterparts in CBOT (dashed curve). The sample period spans September 22, 2004 to December 22, 2018. Source: Wind database.

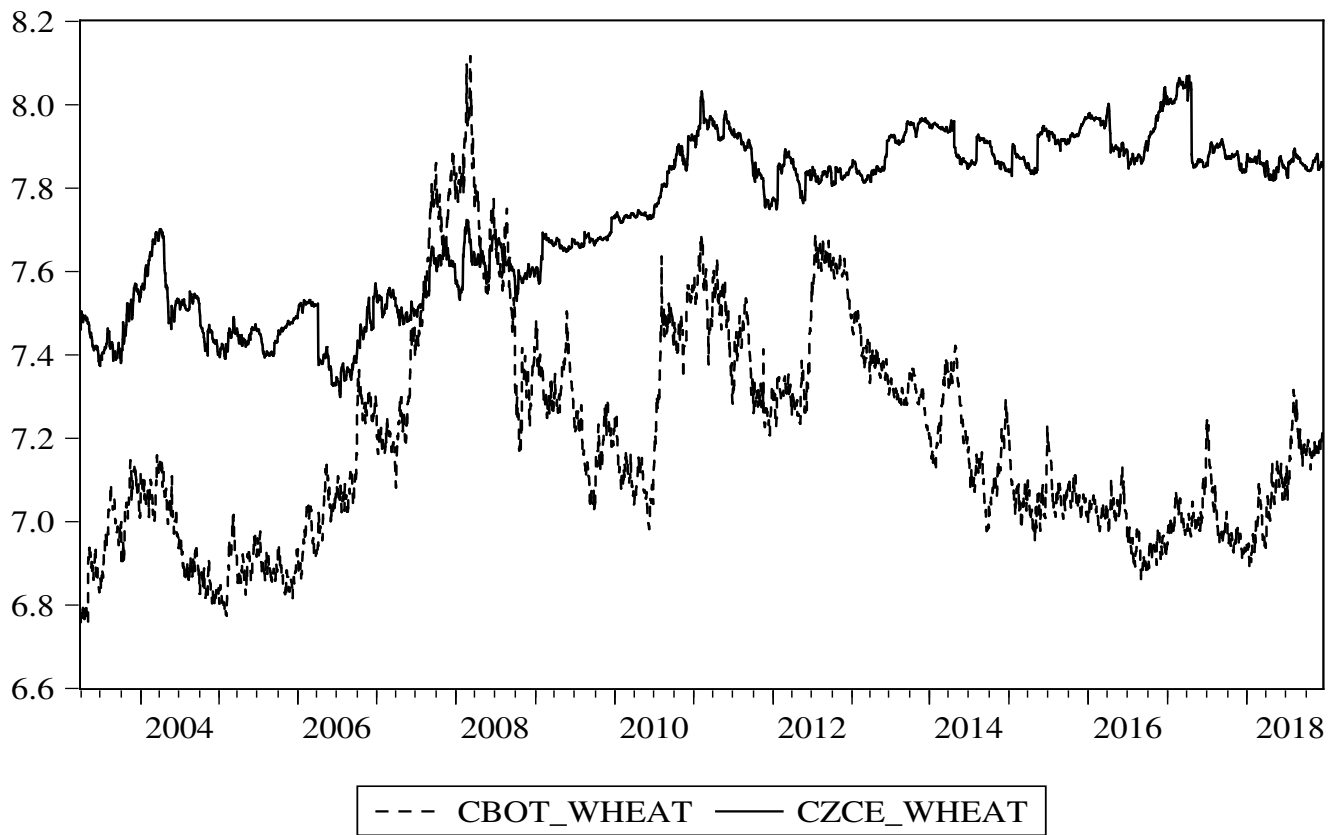

Figure 2: Logarithm of strong gluten wheat futures prices

Notes: This figure plots the logarithmic prices of strong gluten wheat futures in CZCE (solid curve) together with their international counterparts in CBOT (dashed curve). The sample period spans March 28, 2003 to December 22, 2018. Source: Wind database. 


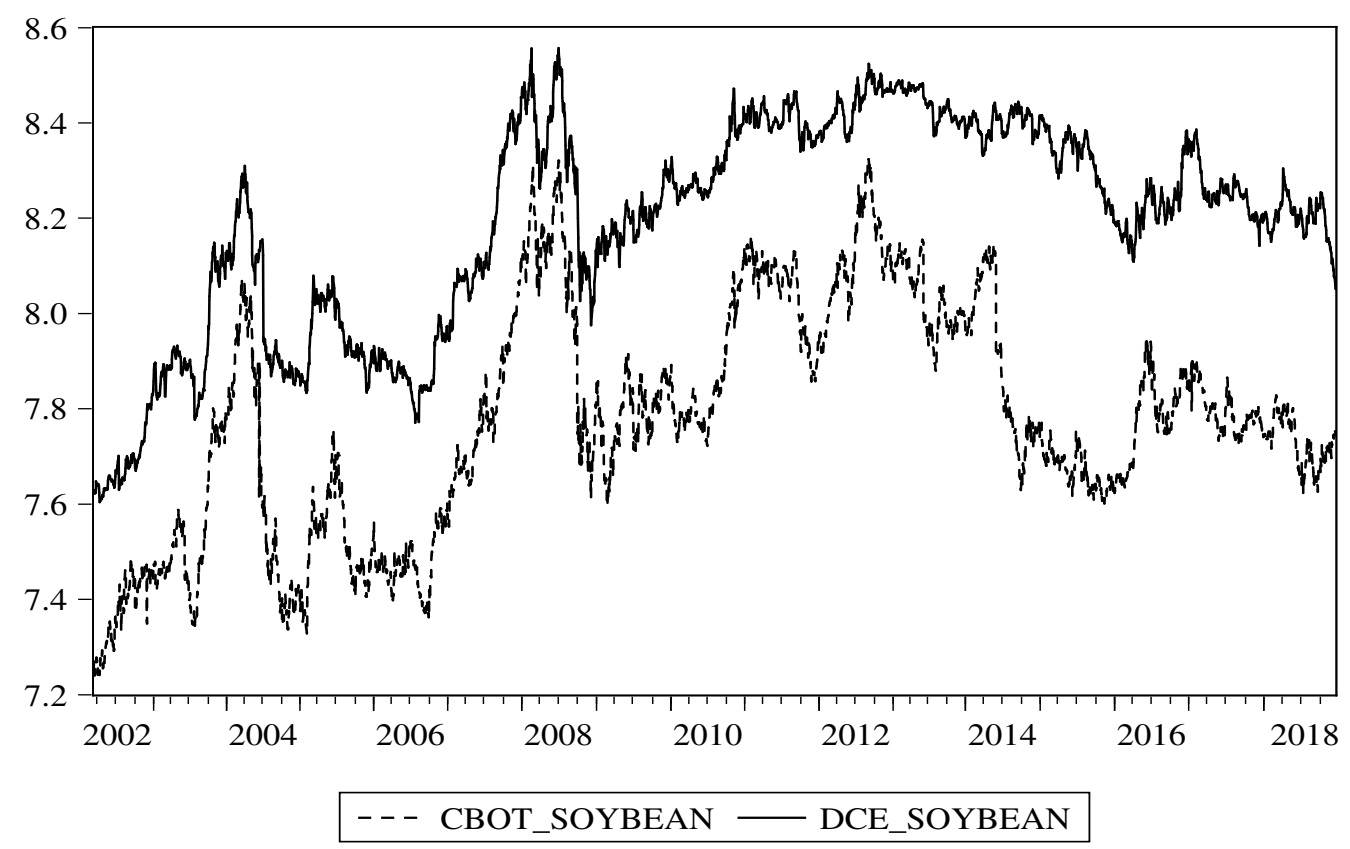

Figure 3: Logarithm of No. 1 soybean futures prices

Notes: This figure plots the logarithmic prices of No. 1 Soybean futures in DCE (solid curve) together with their international counterparts in CBOT (dashed curve). The sample period spans March 15, 2002 to December 22, 2018. Source: Wind database.

drastic fluctuations, because they are by-products. However, soymeal prices were more affected by international transgenic soybean prices. Therefore, fluctuations in soymeal markets were relatively larger than those in No. 1 soybean markets.

Figure 5 shows that prices of cotton futures reached a high point in 2011 and then experienced wild fluctuations. The trading volume of cotton futures in 2011 was second only to crude oil futures globally. From 2012 to 2013, cotton futures prices changed gradually compared to their international counterparts because of the temporary reserve policy. In 2014, China carried out a cotton price reform and handed pricing power to the market. Cotton prices then declined for about two years. For white sugar futures, shown in Figure 6, periodicity is relatively clear because of the characteristic of perennial root growth for sugarcane, the main source of white sugar in China. In the first year, sugarcane is planted and then perennial roots grow for about three years. Therefore, yield increases in the first three years, then decreases in the next three years. Growth habit is an important factor influencing prices of white sugar. Long-term sugar prices decline for three years, then increase over the next three years, in contrast to yield trends. In addition to the effect of growth habit, there is also a temporary reserve policy in the sugar market. Compared to ICE international sugar futures, the Chinese sugar market is less volatile.

Compared to foreign commodities markets, regulation is very common in Chinese markets. Government policies create invisible supports under the market. The OU model has no way to characterize this regulatory phenomenon, while the skew OU model can capture specific regulatory characteristics and preserve mean-reverting features at the same time. This observation forms part of the motivation behind the specific settings of our skew OU model. In the next section below, we briefly introduce 


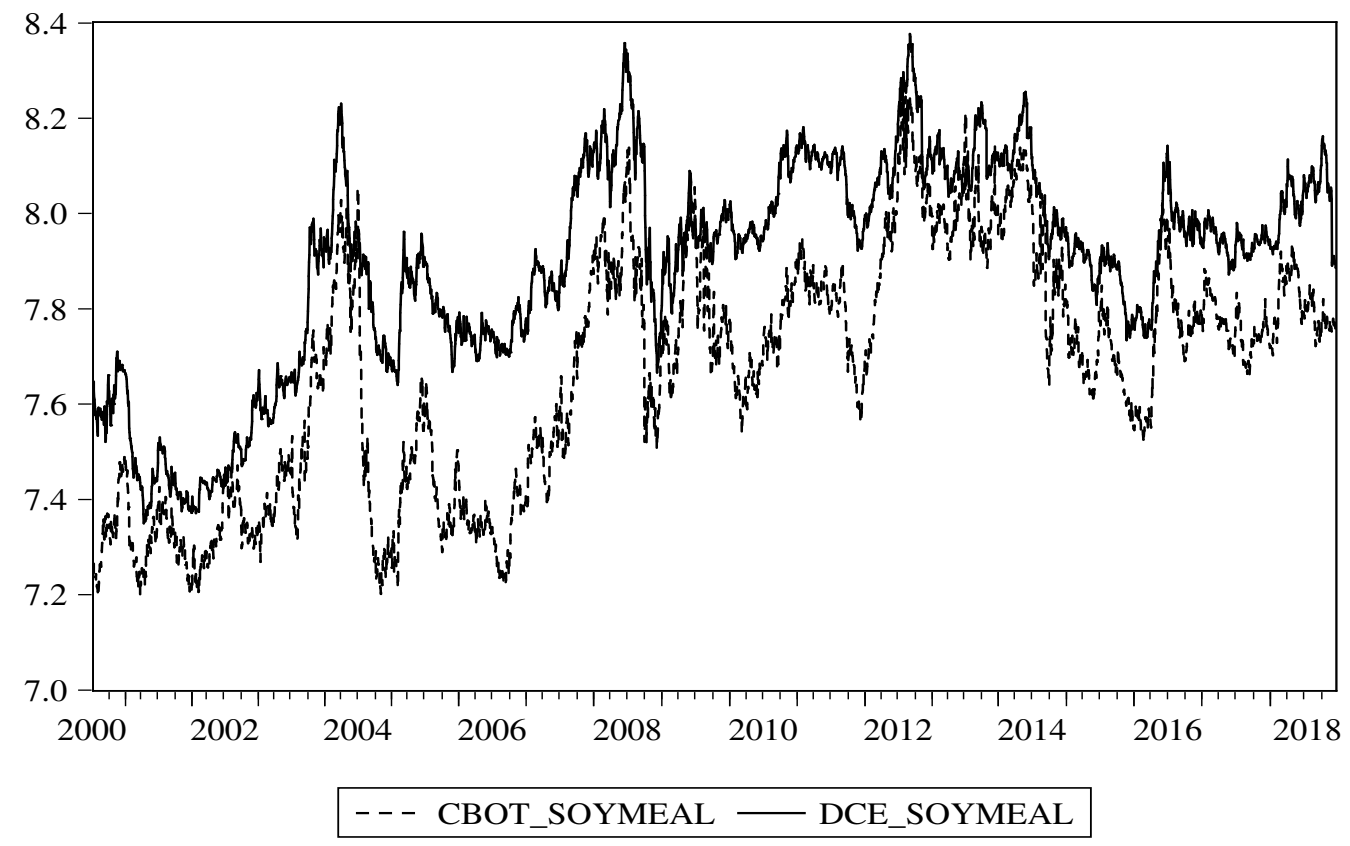

Figure 4: Logarithm of soymeal futures

Notes: This figure plots the logarithmic prices of soymeal futures in DCE (solid curve) together with their international counterparts in CBOT (dashed curve). The sample period spans July 17, 2000 to December 22, 2018. Source: Wind database.

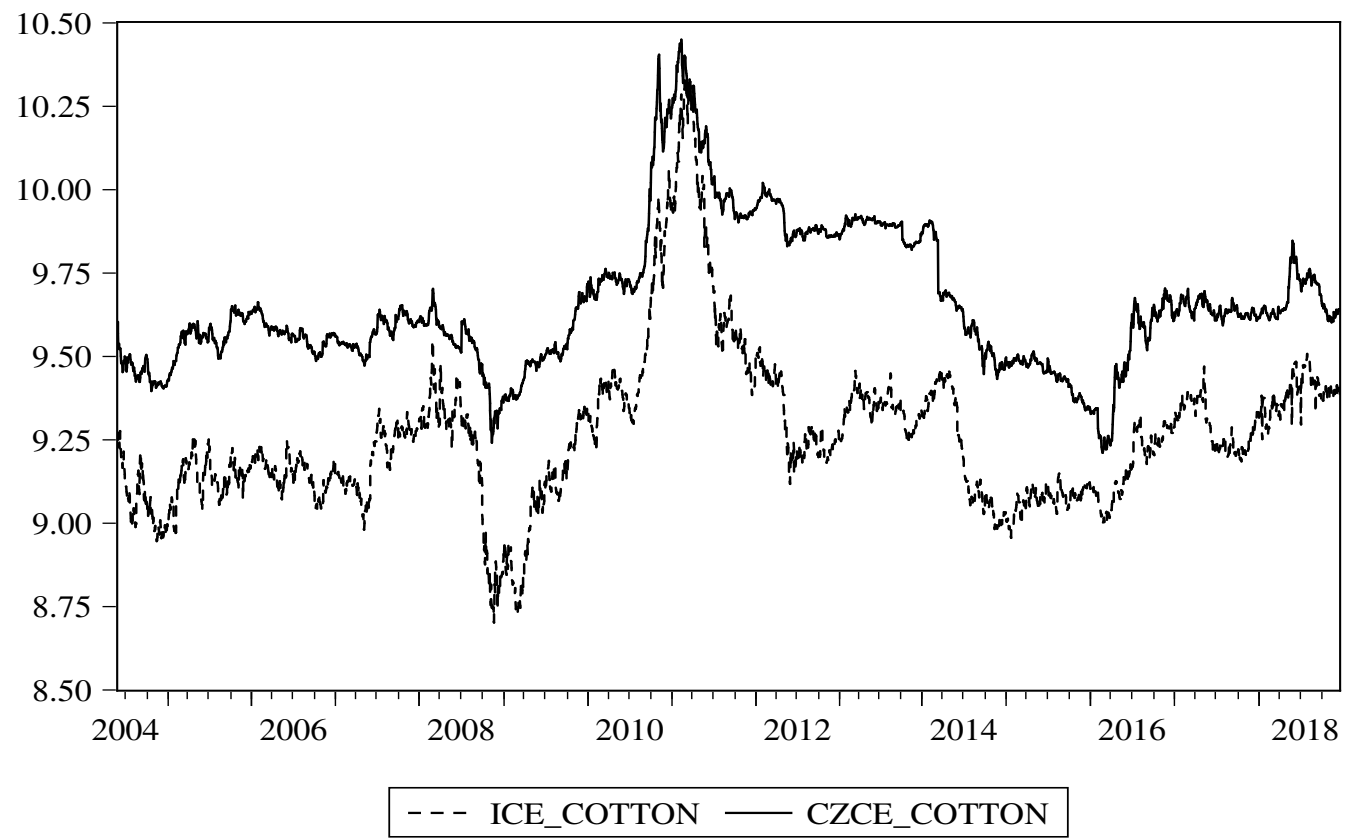

Figure 5: Logarithm of cotton futures prices

Notes: This figure plots the logarithmic prices of cotton futures in CZCE (solid curve) together with their international counterparts in ICE (dashed curve). The sample period spans June 1, 2004 to December 22, 2018. Source: Wind database. 


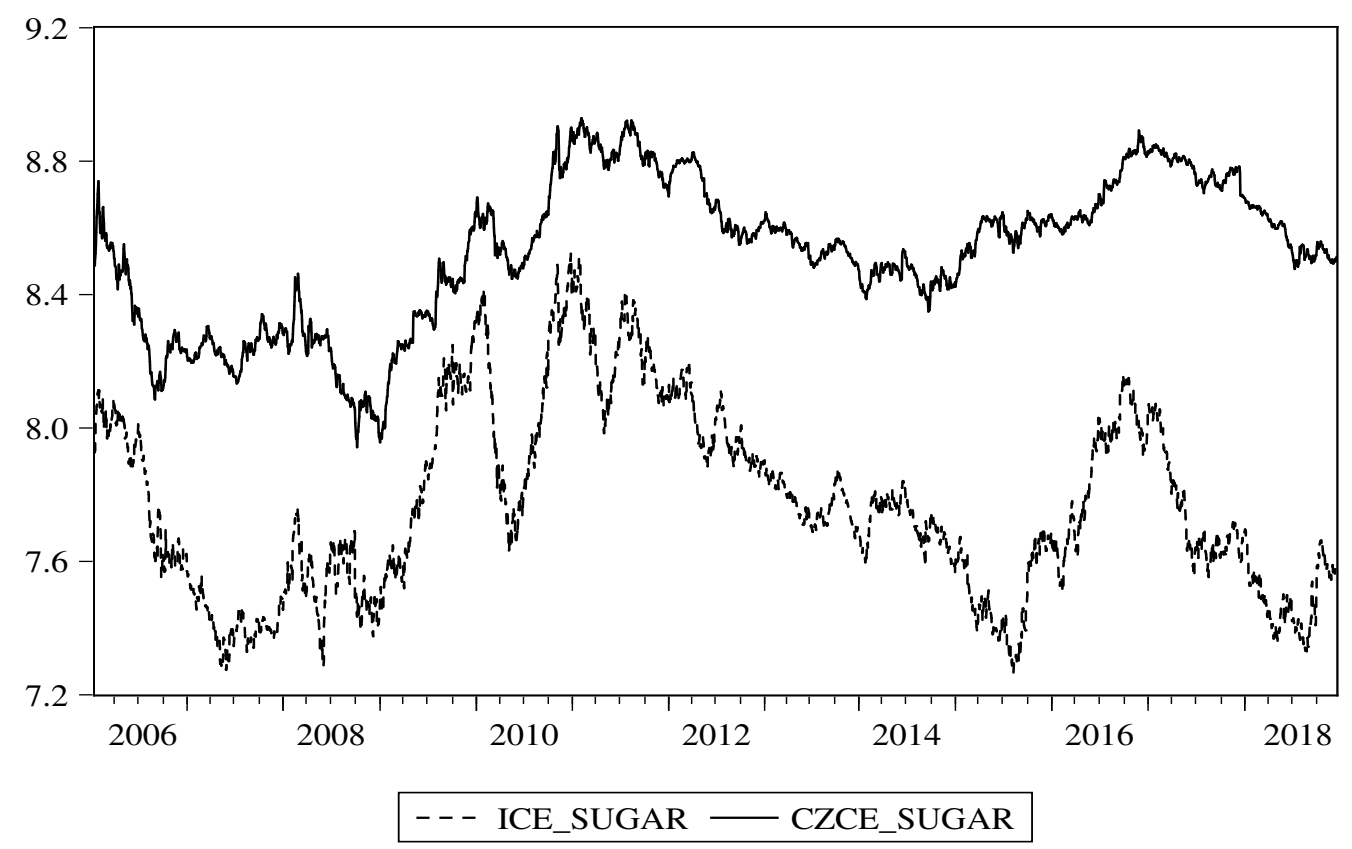

Figure 6: Logarithm of white sugar futures prices

Notes: This figure plots the logarithmic prices of white sugar futures in CZCE (solid curve) together with their international counterparts in ICE (dashed curve). The sample period spans January 16, 2006 to December 22, 2018. Source: Wind database.

the skew OU model.

\section{Skew Diffusion Processes and Parameter Estimation}

This section presents dynamics of the logarithm of agricultural commodity futures prices that follow skew OU processes. When considering model estimation, local time components are hard to address. Therefore, we derive respective transformed processes such that local time terms can be removed and the processes are more tractable. Further, we provide a method to test whether the skew OU process can better fit a given dataset compared to the OU process model. To this end, we conduct a generalized quasi-likelihood ratio test on six agricultural commodity futures where the null hypothesis is that the $\mathrm{OU}$ process is more suitable. In addition, we conduct a significance test to determine whether the skew probability differs from 0.5.

\subsection{Skew OU Process}

Let us fix a probability space $(\Omega, \mathcal{F}, \mathcal{P})$ and an information filtration $\left\{\mathcal{F}_{t}\right\}_{t \geq 0}$. Similar to Schwartz (1997), we assume that commodity futures price $F_{t}$ follows the stochastic process:

$$
d F_{t}=\kappa\left(\mu-\ln F_{t}\right) F_{t} d t+\sigma F_{t} d W_{t} .
$$

This specification of the model can prevent the price from being negative. Based on their model, 
we add a symmetrical local time component to reflect regulatory characteristics:

$$
d F_{t}=\kappa\left(\mu-\ln F_{t}\right) F_{t} d t+\sigma F_{t} d W_{t}+(2 p-1) d \hat{L}_{t}^{F}\left(a^{F}\right)
$$

where $\kappa, \mu, \sigma, p$ and $a^{F}$ are unknown structural parameters. In this setting, $\kappa>0$ is the speed of mean reversion to the unconditional long-term mean price $\mu$, and $\sigma>0$ is the volatility of the process. $\hat{L}_{t}^{F}\left(a^{F}\right)$ is the symmetric local time of the continuous semi-martingale $F=\left\{F_{t}, \mathcal{F}_{t} ; 0 \leq t<+\infty\right\}$ at skew level $a^{F}$, while $p \in(0,1)$ is the skew probability capturing the possibility of upward movement after hitting skew level $a$. More details on the symmetrical local time component are shown in Appendix A.

Defining $X_{t}=\ln F_{t}$ and applying the generalized Itô formula (Revuz and Yor, 1999) on Eq.(2.2), we show that the logarithmic price submits to the skew OU process:

$$
d X_{t}=\kappa\left(\theta-X_{t}\right) d t+\sigma d W_{t}+(2 p-1) d \hat{L}_{t}^{X}(a)
$$

where $\theta=\mu-\frac{\sigma^{2}}{2 \kappa}$ and $a=\ln a^{F}$.

All investigations described in this paper are based on Eq.(2.3) and the empirical research is based on logarithmic price.

\subsection{Methods of Estimation}

This section proposes a method to estimate the skew OU process. The estimation of SBM used by Lejay and Pichot (2012) is not suitable for skew OU processes with non-constant drift. Further, the method introduced by Bardou and Martinez (2010) requires that the coefficients of the OU component are already known and involves a complex multiple integral. This method does not work either. Thus, we put forward a Bayesian approach that can estimate all parameters together and is easily applied to both simulations and real data. Liang et al. (2019) employ a Bayesian method to real gas data.

Since the local time component is difficult to address, before estimation, we define a transformed process $Y_{t}:=G\left(X_{t}\right)$ to remove it using methods introduced by Harrison and Shepp (1981),

$$
G\left(X_{t}\right)= \begin{cases}(1-p)\left(X_{t}-a\right)+a, & \text { if } X_{t} \geq a \\ p\left(X_{t}-a\right)+a, & \text { if } X_{t}<a .\end{cases}
$$

Meanwhile, the inverse transform $X_{t}:=H\left(Y_{t}\right)$ satisfies:

$$
H\left(Y_{t}\right)= \begin{cases}\frac{1}{1-p} Y_{t}-\frac{p}{1-p} a, & \text { if } Y_{t} \geq a \\ \frac{1}{p} Y_{t}-\frac{(1-p)}{p} a, & \text { if } Y_{t}<a\end{cases}
$$

We apply the generalized Itô formula (Revuz and Yor, 1999) to process $Y_{t}$; it then satisfies the following stochastic differential equation:

$$
d Y_{t}= \begin{cases}\kappa\left[(1-p) \theta+p a-Y_{t}\right] d t+(1-p) \sigma d W_{t}, & \text { if } Y_{t} \geq a \\ \kappa\left[p \theta+(1-p) a-Y_{t}\right] d t+p \sigma d W_{t}, & \text { if } Y_{t}<a\end{cases}
$$

We finally obtain the new process $Y_{t}$ without the local time component. The transformation is described in Appendix B. Then, we assume that we are given the data sampled at equally spaced time 
points $t_{1}<\cdots<t_{N+1}$, by setting $y_{t_{i}} \triangleq Y_{t_{i+1}}-Y_{t_{i}}$ and $x_{t_{i}} \triangleq Y_{t_{i}}$, the discretized version of Eq.(2.6) can be expressed as:

$$
y_{t_{i}}= \begin{cases}\kappa[(1-p) \theta+p a] \Delta t-\kappa \Delta t x_{t_{i}}+(1-p) \sigma \sqrt{\triangle t} \epsilon_{t_{i}}, & \text { if } x_{t_{i}} \geq a, \\ \kappa[p \theta+(1-p) a] \Delta t-\kappa \Delta t x_{t_{i}}+p \sigma \sqrt{\triangle t} \epsilon_{t_{i}}, & \text { if } x_{t_{i}}<a .\end{cases}
$$

where $i=1,2, \cdots, N, \triangle t=t_{i+1}-t_{i}$ and $\left\{\epsilon_{t_{i}}\right\}_{i=1}^{N}$ are independent and standard normally distributed. The discretized version is similar to the threshold autoregressive model, which is usually estimated via Bayesian estimation method. We eliminate local time items via transformation. Only when a set of skew levels and skew probability values are given in advance can we achieve this goal. Traditional estimation methods are troublesome in this case, while Bayesian estimation method has several advantages.

Accordingly, a Bayesian estimation method is adopted. First, owing to the work described above, we obtain the likelihood function of Eq.(2.7):

$$
\begin{aligned}
L(X \mid \Theta)= & \left(\frac{1}{(1-p) \sqrt{2 \pi \sigma^{2} \triangle t}}\right)^{n_{1}} \exp \left\{-\frac{1}{2(1-p)^{2} \sigma^{2} \triangle t}\right. \\
& \left.\times \sum_{i \in N_{1}}\left[y_{t_{i}}+\kappa \triangle t x_{t_{i}}-\kappa \triangle t((1-p) \theta+p a)\right]^{2}\right\} \\
& \times\left(\frac{1}{p \sqrt{2 \pi \sigma^{2} \triangle t}}\right)^{n_{2}} \exp \left\{-\frac{1}{2 p^{2} \sigma^{2} \triangle t}\right. \\
& \left.\times \sum_{i \in N_{2}}\left[y_{t_{i}}+\kappa \triangle t x_{t_{i}}-\kappa \triangle t(p \theta+(1-p) a)\right]^{2}\right\},
\end{aligned}
$$

where $\Theta$ is the set of five parameters $\left\{\kappa, \theta, \sigma^{2}, a, p\right\}$ for the skew OU process, and $X$ represents the data set. $N_{1} \triangleq\left\{i: i=1, \cdots, N, a \leq x_{t_{i}}\right\}$ and $N_{2} \triangleq\left\{i: i=1, \cdots, N, x_{t_{i}}<a\right\}$ where $n_{1}$ and $n_{2}$ are the amounts of $i$ included in the sets $N_{1}$ and $N_{2}$, respectively.

Then, we obtain the Bayesian estimates. The joint distribution of parameters is difficult to address. Therefore, we use the Gibbs sampling method to simulate the conditional posterior distributions of parameters and loop 1000 times. Discarding the first 200 sampling values, we get parameter estimations through taking the average of the last 800 sampling values. The standard deviation of the parameter equals that of the last 800 sampling values. To this end, we first define the prior distributions of $\Theta$ by choosing conjugate distributions. We take the prior distribution of $\kappa$ and $\theta$ to be an independent normal distribution $\mathcal{N}\left(\mu_{\kappa}, \sigma_{\kappa}^{2}\right)$ and $\mathcal{N}\left(\mu_{\theta}, \sigma_{\theta}^{2}\right)$. The prior of $\sigma^{2}$ is assumed to be an inverse gamma distribution $\mathcal{I G}\left(\alpha_{\sigma}, \lambda_{\sigma}\right)$. Also, we consider the prior of $a$ and $p$ to follow discrete uniform distributions on sets $\left\{a_{1}, \cdots, a_{n}\right\}$ and $\left\{p_{1}, \cdots, p_{n}\right\}$. Further, we derive the conditional posterior distributions of $\Theta$ in Appendix C. From the above analysis, we use the Gibbs sampler to simulate the conditional posterior distributions. By generating a Markov chain of parameters, we obtain the estimations of $\Theta$. We also detect convergence through a convergence diagnostic (CD) test as in Appendix D.

\subsection{Significance Test of Skew Probability}

We address whether the estimate of the skew probability $\hat{p}$ is significantly different from 0.5 . Skew level is significant only when the skew probability estimate is significantly different from 0.5 . This 
amounts to testing $H_{0}: \kappa=\kappa_{1}, \theta=\theta_{1}, \sigma=\sigma_{1}, a=a_{1}, p=0.5$ against $H_{1}: \kappa=\kappa_{1}, \theta=\theta_{1}, \sigma=\sigma_{1}$, $a=a_{1}, p=p_{1}$. Parameters in the hypothesis are all estimated via the skew OU model.

Under the null hypothesis, the log-likelihood function is:

$$
l\left(H_{0}\right)=-\left\{\frac{N}{2} \cdot \ln \left(\sigma_{1}^{2} \Delta t\right)+\frac{\sum_{i=1}^{N}\left(y_{t_{i}}+\kappa_{1} \theta_{1} \Delta t x_{t_{i}}-\kappa_{1} \Delta t\right)^{2}}{2 \sigma_{1}^{2} \Delta t}\right\}
$$

Under the alternative hypothesis, the log-likelihood function is:

$$
\begin{aligned}
l\left(H_{1}\right)= & -\left\{\frac{n_{1}}{2} \cdot \ln \left[\left(1-p_{1}\right)^{2} \sigma_{1}^{2} \triangle t\right]+\frac{\sum_{i \in N_{1}}\left\{y_{t_{i}}+\kappa_{1} \triangle t x_{t_{i}}-\kappa_{1} \triangle t\left[\left(1-p_{1}\right) \theta_{1}+p_{1} a_{1}\right]\right\}^{2}}{2\left(1-p_{1}\right)^{2} \sigma_{1}^{2} \triangle t}\right\} \\
& -\left\{\frac{n_{2}}{2} \cdot \ln \left(p_{1}^{2} \sigma_{1}^{2} \triangle t\right)+\frac{\sum_{i \in N_{2}}\left\{y_{t_{i}}+\kappa_{1} \triangle t x_{t_{i}}-\kappa_{1} \triangle t\left[p_{1} \theta_{1}+\left(1-p_{1}\right) a_{1}\right]\right\}^{2}}{2 p_{1}^{2} \sigma_{1}^{2} \triangle t}\right\},
\end{aligned}
$$

where $n_{1}+n_{2}=N$.

The plausibility of the hypotheses can then be evaluated by:

$$
\lambda(X)=2\left[l\left(H_{1}\right)-l\left(H_{0}\right)\right]
$$

where $\mathrm{X}$ is the sample data.

To test the significance of the estimated value of the skew probability, it is necessary to know the distribution of $\lambda(X)$ under the null hypothesis. We use the bootstrap procedure to get the distribution when the skew probability equals 0.5. Under the null hypothesis, we first generate simulated sample $X^{*}$ from $d X_{t}=\kappa_{1}\left(\theta_{1}-X_{t}\right) d t+\sigma_{1} d W_{t}+(2 \cdot 0.5-1) d \hat{L}_{t}^{X}\left(a_{1}\right)$ and then estimate the parameters via Bayesian methods. Finally, we obtain the test statistics $\lambda\left(X^{*}\right)$. We repeat this procedure 10,000 times and get 10,000 $\lambda\left(X^{*}\right)$ to estimate the distribution of $\lambda(X)$ under the null hypothesis.

Thus, we observe that when skew probability equals $0.5, l\left(H_{1}\right)$ will not reduce to $l\left(H_{0}\right)$. Therefore, we cannot conclude that if skew probability $p$ is not significantly different from 0.5 , the values of $l\left(H_{1}\right)$ and $l\left(H_{0}\right)$ will be close. As a result, extreme small-probability cases on both sides are taken as rejection domains. If the $p$-value of $\lambda(X)$ is too small for a particular level of significance, we can reject the null hypothesis and illustrate that the skew probability is significant.

\subsection{Goodness-of-fit Test}

Because the OU model can be regarded as a special case of the skew OU model by setting $p$ equal to 0.5 , it is natural to ask whether the skew OU model can do a better job at fitting the dynamics of Chinese agricultural commodity futures prices. In other words, are there truly skew phenomena in the Chinese agricultural commodity futures market? This amounts to testing $H_{0}: \kappa=\kappa_{0}, \theta=\theta_{0}, \sigma=\sigma_{0}$, $p=0.5$ against $H_{1}: \kappa=\kappa_{1}, \theta=\theta_{1}, \sigma=\sigma_{1}, a=a_{1}, p=p_{1}$. Parameters in the null hypothesis are estimated via OU model while those in the alternative hypothesis are estimated via skew OU model.

Under the null hypothesis, the log-likelihood function is:

$$
l\left(H_{0}\right)=-\left\{\frac{N}{2} \cdot \ln \left(\sigma_{0}^{2} \Delta t\right)+\frac{\sum_{i=1}^{N}\left(y_{t_{i}}+\kappa_{0} \theta_{0} \Delta t x_{t_{i}}-\kappa_{0} \Delta t\right)^{2}}{2 \sigma_{0}^{2} \Delta t}\right\}
$$

Under the alternative hypothesis, the log-likelihood function $l\left(H_{1}\right)$ is the same as Eq.(2.10). The 
plausibility of the hypotheses can then be evaluated by:

$$
\lambda(X)=2\left[l\left(H_{1}\right)-l\left(H_{0}\right)\right],
$$

where $\mathrm{X}$ is the sample data.

Note that the distribution of $\lambda(X)$ under the null hypothesis is difficult to calculate. Thus, we use the bootstrap procedure to determine whether to reject the null hypothesis. Under the null hypothesis, we first generate simulated sample $X^{*}$ from the OU process by setting the parameters at the maximum likelihood estimates, and then estimate the parameters using Bayesian methods. In this way, we obtain the test statistics $\lambda\left(X^{*}\right)$. We repeat this procedure 10,000 times and obtain 10,000 $\lambda\left(X^{*}\right)$ to estimate the distribution of $\lambda(X)$ under the null hypothesis.

As illustrated in subsection 3.2, because local time is hard to address directly, we do not tackle the original process with the local time component. Instead, we transform the process into a tractable piecewise diffusion without local time. Therefore, before we estimate the skew OU process, we need to perform a piecewise transformation on the data. As a result, even when skew probability equals $0.5, l\left(H_{1}\right)$ will not reduce to $l\left(H_{0}\right)$. Therefore, similar to subsection 3.3 , we choose extremely low probability cases on both sides as rejection domains. If the $p$-value of $\lambda(X)$ is too small, we can reject the null hypothesis and illustrate that the skew OU model is superior to the OU model.

\section{Empirical Results}

Tables 3-8 list parameter estimation results of mean-reversion coefficients, long-term mean, volatility, skew levels, skew probabilities, as well as corresponding $p$-values and $p$-values of generalized quasilikelihood ratio tests for the skew OU model. In addition, we report OU estimates. The model is separately estimated for each future and all calculations are realized via $\mathrm{R}$ software. Considering that outliers have a great impact on estimation results, we use the 3-sigma criterion to eliminate outliers. In addition, because the data in the first year are incomplete and the quality of data when futures are just launched is not sufficient, we estimate all parameters from the second year of the sample period. Table 3 provides estimation results with respect to corn futures while Tables 4-8 provide similar results for strong gluten wheat, No. 1 soybean, soymeal, cotton, and white sugar, respectively.

\subsection{Grain Futures Market}

Mean-reversion coefficients for corn futures vary significantly from year to year ${ }^{8}$. Years 2012 and 2014 had much stronger mean-reversion parameters, which means that it took less time to go from the starting value to the long-term mean. Values of parameters are not stable across time, indicating that it is useful to estimate parameters by year, or in the future, allow parameters to be time-variant. Among all parameters, skew levels and skew probabilities are of great interest. In addition, among all estimates, results significant at the $10 \%$ level interest us most. When logarithmic prices hit the skew level, in addition to the fact that the mean reversion term will pull the price toward the long-term level, the market will exert additional forces to cause the price to have a higher probability of reverting. Table 3 shows that skew probabilities are significantly different from 0.5 in almost half the samples for corn futures at the $10 \%$ significance level. In 2005, 2006, 2008, and 2009, skew levels behaved like

\footnotetext{
${ }^{8}$ According to the nonparametric model introduced by Fan et al. (2003), $\kappa$ also varies by year.
} 
potential supporting lines, with corresponding skew probabilities greater than 0.5. In 2013, 2014, and 2015 , they acted as lines of pressure with corresponding skew probabilities smaller than 0.5. Figure 7 illustrates this in a more intuitive way. When skew probability is clearly different from 0.5 , we label its corresponding skew level in Figure 7. If the skew probability is larger (smaller) than 0.5, we treat its corresponding skew level, marked in red (green) in Figure 7, as a support (resistance) line.

Why do skew phenomena exist? Skew level formation is likely to be associated with government intervention. For the corn market, a specific purchase price is announced every year before planting. Annual purchases of grain by state-owned grain enterprises account for at least $40 \%$ of the newly planted grain, which enables the government to control the grain supply, enabling a substantial impact on the purchase market. Annual sales by state-owned grain enterprises also account for significantly more than $40 \%$ of grain circulation. Consequently, such enterprises are able to effectively influence prices in the grain sales market ${ }^{9}$. Despite the fact that government intervention is implemented mainly in the spot market, it is common knowledge that futures and spot prices are highly correlated. Therefore, factors affecting spot prices will also influence futures prices. Skew levels estimated by the skew OU model behave like price levels of temporary reserves in the corn market. For example, the average price for temporary purchase and stocking of corn in 2008 and 2009 was approximately 1,500 RMB per ton; in 2013 and 2014 it was approximately 2,240 RMB per ton, while in 2015 it was approximately $2,000 \mathrm{RMB}$ per ton ${ }^{10}$, very close to the skew levels. As the skew probability deviates from 0.5 , the skew level is harder to break through, which indicates that government intervention policy is effective. For example, the skew probability of corn futures in 2009 was 0.8080 , which means that when prices of corn futures hit the skew level, there was an $80.80 \%$ probability of a future rise. The government implemented a temporary reserve policy for corn from the end of 2008, which played an important role in supporting the market.

The original intention of the policy was to encourage the planting of corn to ensure national food security. However, international agricultural prices, including corn, plunged after 2011, while domestic corn prices have risen yearly. That left domestic stocks piling up, resulting in a glut. The import volume of corn in China was relatively restricted and differences between internal and external prices made domestic corn enterprises come under high cost pressures. Multiple factors led to a higher probability of declining corn prices, as reflected in low skew probabilities in 2013 and 2014. As shown in Figure 1, since 2012, international corn prices have peaked and entered a long bear market, while domestic corn prices remained at a relatively stable level. Although government policy supported the market, the true dynamics meant potential downward pressure, as reflected by skew probabilities of less than 0.5. Skew phenomena were not significant from 2010 to 2012. According to the Ministry of Agriculture, the amount of corn reserve was close to zero in these three years. Therefore, government intervention in this case was limited. In 2015, skew probability was 0.1621 , far less than 0.5 . When prices of corn futures hit the skew level, there was only a $16.21 \%$ probability of a price increase. The government for the first time lowered purchase prices in 2015, cutting them about $12 \%$. This event was regarded as a signal that the government would implement temporary reserve policy reform. In addition, previous overstocking put pressure on prices. The total stock of state reserves reached 236 million tons, and the inventory-to-consumption ratio reached a new high of $106 \%$ in $2015^{10}$. Government policy no longer acted as the supporting power but as a signal that

\footnotetext{
${ }^{9}$ Information is from China Grain Development Report.

${ }^{10}$ Data are from futures company.
} 
corn prices would decline in the future. After 2015, there occurred no significant skew phenomena in corn futures markets. In March 2016, the government abolished the policy of temporary reserves and proposed a new policy of market-based purchases and subsidies. The degree of marketization of corn increased, and the government began to weaken macro-regulation. Such changes in policy can be detected in the disappearance of skew phenomena.

If skew probability is close to 0.5 , breaking through potential boundaries is acceptable. The government believed price fluctuations could be addressed through market mechanics. As reported in Table 3, skew probabilities for 2006 and 2008 were very close to 0.5 , which meant that corresponding skew levels were soft. Once the price hits the skew level, the probability of going up or down is about the same. Under this circumstance, government intervention is less effective.

The CD statistics in Table 3 show that the Markov chains attain convergence, indicating that the Markov Chain Monte Carlo (MCMC) estimations are effective. The generalized quasi-likelihood ratio test is statistically significant at the $10 \%$ level, indicating that the skew OU model is superior to the OU model when skew phenomena are significant in corn futures markets. In addition, we report the estimation results of maximum likelihood estimation (MLE) for the OU model, with parameters $\kappa, \theta$ and $\sigma$. Parameters $\kappa$ in the OU model are not significant in most years. These results show that the skew OU model is superior to the OU model when modeling the Chinese corn futures market with government controls.

Skew phenomenon is significant for more than half of samples in the strong gluten wheat market. For strong gluten wheat futures, 2008, 2009, 2010, 2012, and 2015 formed strong levels of support, while 2004, 2005, 2014, and 2017 showed significant resistance. These phenomena are also observed in Figure 8. The government first implemented a floor purchase price policy for wheat in 2006. Although the policy was first set in 2005, it was not launched that year. After implementation of the policy, wheat prices showed a clear upward trend. The minimum purchase price level of wheat increased steadily from 2006 to 2014, with a cumulative increase of over $70 \%{ }^{11}$. From parameter estimation results of the skew OU model, we find that it took several years for this policy to work. Since 2008, the wheat market showed significant supporting levels in four years, except for 2011. According to data from the China Statistical Yearbook, since the start of the minimum purchase policy, except for 2005 and 2011, wheat purchases were launched in all other years, with a cumulative purchase of more than 300 million tons. In 2011, the market price of wheat was higher than the minimum purchase price, so the government did not implement a minimum-price purchase. The skew OU model can exactly detect such a change in policy. The quantity of the minimum purchase also affected the efficiency of government policy. In 2013, this quantity accounted for only about $6 \%$ of total production. Such effects are also demonstrated by no significant skew phenomena in 2013. In other years, the percentage was more than $20 \%$ and approached $40 \%$. As a whole, government policies could have a relatively important impact on the market. Skew levels in 2004, 2005, 2014, and 2017 acted as pressure levels. The increase in yields, imports, and local reserves of wheat in 2004 and 2005 resulted in higher supply that did not match demand. Thus, wheat prices showed downward pressure. Skew levels may act as psychological price levels for farmers and investors. Psychological barriers can be regarded as price levels that are psychologically important to the extent that the market finds it difficult to reach that

\footnotetext{
${ }^{11}$ Data are from the China Food Network.
} 
Table 3: Parameter estimation results of skew OU model in corn futures market

\begin{tabular}{|c|c|c|c|c|c|c|c|c|c|c|}
\hline \multirow{2}{*}{ Year } & \multicolumn{7}{|c|}{ Skew OU estimates } & \multicolumn{3}{|c|}{ OU estimates } \\
\hline & $\hat{\kappa}$ & $\hat{\theta}$ & $\hat{\sigma}$ & $\hat{a}$ & $\hat{p}$ & $p$-value $^{1}$ & $p$-value ${ }^{2}$ & $\hat{\kappa}_{0}$ & $\hat{\theta}_{0}$ & $\hat{\sigma}_{0}$ \\
\hline \multirow{3}{*}{2005} & & & & & & & & 7 & 55706 & 00705 \\
\hline & & & & & & & & & & \\
\hline & [1.2069] & {$[-0.3892]$} & {$[0.4217]$} & {$[0.4347]$} & {$[-0.9321]$} & {$[395.2732]$} & {$[385.1865]$} & & {$[1111.6837$} & \\
\hline \multirow{3}{*}{2006} & & & & & 0.5778 & & & -0.9 & 7.2564 & \\
\hline & $(0.4745)$ & & & & $27)$ & & & $(2.3550)$ & 5) & \\
\hline & {$[-0.4329]$} & {$[0.6808]$} & {$[0.1591]$} & {$[1.0118]$} & {$[0.4559]$} & {$[298$} & {$[287.4541]$} & & 1041.8828 & \\
\hline \multirow{3}{*}{2007} & 3.4707 & & & 1 & 89 & & & 3.568 & 1.0 & 0. \\
\hline & $(0.4$ & & & & & & & 50) & & \\
\hline & {$[-0.7253]$} & [1.3323] & {$[-1.3011]$} & {$[-0.6394]$} & {$[1.5810]$} & {$[39$} & {$[39$} & & {$[1062$} & \\
\hline \multirow{3}{*}{2008} & & & & & & & & & 7.3 & \\
\hline & $(0$. & 4) & $0)$ & $(0$ & 21) & & & $(1.5681)$ & 1 & \\
\hline & {$[0.5$} & {$[-0$} & {$[0.4$} & {$[-1$} & {$[-0$} & & & & & \\
\hline \multirow{3}{*}{2009} & & & & & & & & & & \\
\hline & $(0.3986)$ & 8) & 6) & $(c$ & & $(9:$ & & $(1$. & & \\
\hline & {$[-0.1$} & {$[1]$.} & {$[-0$} & {$[-0$} & 61] & & & & & \\
\hline \multirow{3}{*}{2010} & & & & & & & & & & \\
\hline & $(0$. & & & & & & & & & \\
\hline & {$[0.2$} & {$[0.2471]$} & {$[1$.} & & {$[1.3382]$} & $209]$ & & & & \\
\hline \multirow{3}{*}{2011} & & & & & & & & & & \\
\hline & & & & & & & & & & \\
\hline & {$[-1.0$} & [1.3860] & $22]$ & {$[-0$} & $527]$ & 39 & & & & \\
\hline \multirow{3}{*}{2012} & & & & & & & & & 77 & \\
\hline & $(0.6$ & & & & & & & & & \\
\hline & {$[-1.0242]$} & {$[0.4962]$} & $96]$ & {$[-0$} & $82]$ & & & & & \\
\hline \multirow{3}{*}{2013} & & & & & & & & & & \\
\hline & $(0.2913)$ & & & & & & & & & \\
\hline & & & & & & & & & {$[1277$.} & \\
\hline \multirow{3}{*}{2014} & & & & & & & & & & \\
\hline & $(0$. & 6) & 4) & & 20) & (10 & & & & \\
\hline & & & & & & & & & & \\
\hline \multirow{3}{*}{2015} & & & & & & & & & 8.7 & \\
\hline & $(0$. & & & & & & & 59) & & \\
\hline & {$[0$.} & {$[0$.} & {$[-1.0590]$} & {$[-1$} & {$[-0.8361]$} & [399.9555] & {$[39$} & & {$[1101$} & \\
\hline \multirow{3}{*}{2016} & & & & & & 02382 & & & 7.3 & \\
\hline & & & & & & & & & & \\
\hline & [1.4798] & {$[0.0562]$} & {$[-0.1783]$} & [0.8398] & {$[0.3806]$} & {$[398$} & 568] & & 1002.5336 & \\
\hline \multirow{3}{*}{2017} & & & & & & & & 0.1 & 7.4 & \\
\hline & $(0.3259)$ & $(0.00$ & 36) & $(0$. & $(0.0906)$ & $(34$ & 15) & $(2.2217)$ & $(0.0244)$ & $(0.003$ \\
\hline & {$[0.4709]$} & {$[0.3505]$} & {$[-0.7824]$} & {$[0$.} & {$[0.4539]$} & [418.0268] & {$[417$} & & {$[1109.3311]$} & \\
\hline \multirow{3}{*}{2018} & 3.3583 & & & & & & & & & \\
\hline & $(0.4560)$ & $(0.0040)$ & & & $(0.2436)$ & $(350.1426)$ & $(339.8636)$ & $(2.12$ & & $(0.00$ \\
\hline & {$[-0.3057]$} & {$[-0.1186]$} & {$[-0.1054]$} & {$[-0.0789]$} & {$[-1.6367]$} & {$[389.1472]$} & [381.9090] & & {$[1122.4319$} & \\
\hline
\end{tabular}

Notes: Columns (2) to (6) report estimation results based on skew OU model via Bayesian methods. Standard errors are reported in parentheses. CD statistics are reported in square brackets. Column (7) reports $p$-value ${ }^{1}$ of whether the estimator of the skew probability is significantly different from 0.5 . Column (8) reports $p$-value ${ }^{2}$ of the generalized quasi-likelihood ratio test for the null hypothesis that the OU model is more suitable than the skew OU model. Corresponding test statistics are reported in parentheses. To save space, the critical values at the significance level of $10 \%$ used are reported in square brackets. Columns (9) to (11) report estimation results based on the OU model via MLE methods. Standard errors are reported in parentheses. Values of the maximized log-likelihood function are reported in square brackets. The estimation period is based on daily observations from January 1, 2005 to December 22, 2018. 


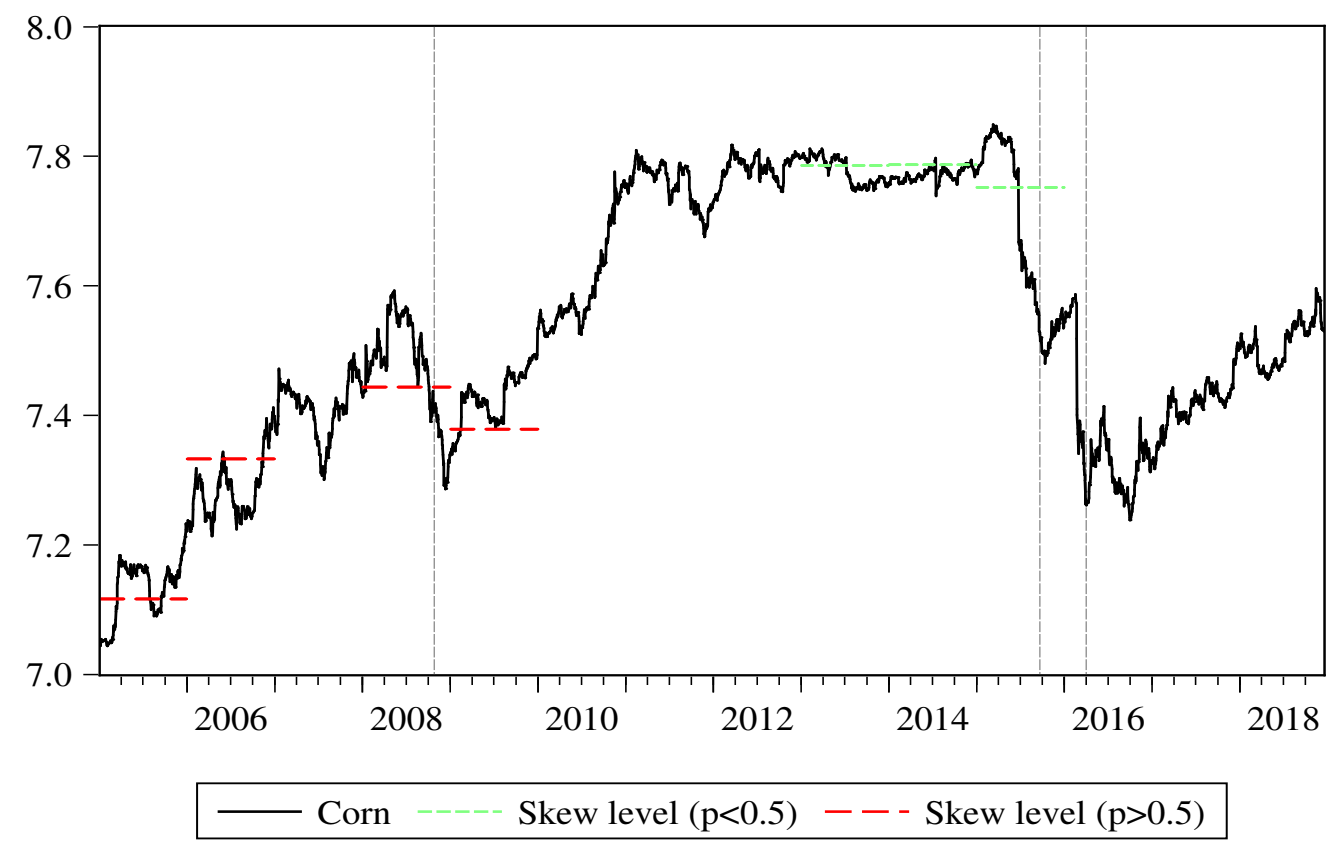

Figure 7: Skew phenomena in corn futures market

Notes: This figure shows logarithmic prices of corn futures (solid curve) with skew levels where skew probabilities are significantly larger than 0.5 (horizontal red dashed lines) and skew levels where skew probabilities are significantly smaller than 0.5 (horizontal green dashed lines). Vertical dashed lines illustrate policy-change split points. The first one marks government implementation of a temporary reserve policy for corn from October 20, 2008, which played an important role in supporting the market. The second one shows the government for the first time lowering purchase prices on September 17, 2015, cutting them about $12 \%$. This event was regarded as a signal that the government would implement temporary reserve policy reform. The last one signals that on March 28, 2016, the government abolished the policy of temporary reserves and proposed a new policy of market-based acquisitions and subsidies. The sample period extends from January 1, 2005 to December 22, 2018.

price during upturns or downturns. Several researchers provide empirical evidence of psychological barriers in gold (Aggarwal and Lucey, 2007) and energy derivatives markets (Dowling et al., 2016; Narayan et al., 2011). Futures prices are affected by a wide variety of behavioral biases, according to behavioral finance (Hirshleifer, 2001). Formation of psychological barriers is likely to be caused by an influx of sell or buy orders, reflecting heterogeneous expectations on specific events. Consistent with the literature, our findings suggest the presence of psychological barriers in agricultural commodity futures markets ${ }^{12}$.

Wheat prices in 2014 ended lower after initially rising in the first half of the year. Under the state policy of a minimum purchase price, a specific price level is set in advance. Once prices increase too fast and remain sustainably above the minimum purchase price, this policy would stop. The aim of the minimum purchase policy was to stabilize prices, not to raise prices. The minimum purchase price was not exactly the actual purchase price in the market; the aim was to play a supporting role when market prices fell excessively. The skew probability in 2014 was far lower than 0.5 , which

\footnotetext{
${ }^{12}$ We conducted tests according to the concept of cluster behavior derived by (Avery and Zemsky, 1998; Westerhoff, 2003).
} 
reflected such phenomena. The government bought a large amount of wheat in 2016 to support the market, and wheat became overstocked in 2017, which put pressure on price dynamics. The National Development and Reform Commission (NDRC) cut the minimum purchase price for wheat for the first time in 12 years in 2017. Such changes sent a signal that the government had begun to reform and destock, which was negative market news. The skew probability in 2017 was far less than 0.5, which reflected this phenomenon from another perspective. The minimum purchase price of wheat was slightly reduced for 2018 and 2019. Imported wheat had obvious cost advantages, and wide price differences between domestic and international wheat put pressure on the support policy. Such price differences are observed in Figure 2. Imported wheat was favored in the domestic market, and the import volume of wheat maintained a high level. This is why skew phenomena cannot be detected in 2018. A reasonable explanation for this is that the power of government intervention could not exceed the power of markets.

The CD statistics in Table 4 show that the Markov chains converge, which demonstrates the effectiveness of MCMC estimation. The generalized quasi-likelihood ratio test is significant at the $10 \%$ level, indicating that the skew OU model is superior to the OU model when skew phenomena are significant in strong gluten wheat futures markets. In addition, we report the estimation results of MLE for the OU model, with parameters $\kappa, \theta$, and $\sigma$. Parameters $\kappa$ in the OU model are not always significant for the whole sample. Such results show that the skew OU model is superior to the OU model when modeling the Chinese strong gluten wheat futures market with regulation.

\subsection{Oilseed Futures Market}

The estimation results for No. 1 soybean futures are tabulated in Table 5 . The speed of mean reversion shows great variation in No. 1 soybean markets. The mean-reversion coefficients of No. 1 soybean in 2011 and 2014 were greater than 8, indicating that, from the starting point to the long-term mean, the process took less time. Other parameters are similar in magnitude. Skew probability is different from 0.5 at the $10 \%$ significance level in almost half of samples for No. 1 soybean futures. The skew probability was 0.1646 in 2004, indicating that prices were more likely to go down upon hitting the skew level. This phenomenon was consistent with the 2004 soybean crisis. Skew levels may not only reflect government intervention, but also the impact of specific events. In this case, skew levels acted as psychological levels. We can explain this from the perspective of behavioral finance as mentioned above in subsection 4.1. There existed potential strong supporting levels in 2007, 2009, and 2010, when skew probability was greater than 0.5. As shown in Figure 9, prices were at a relatively low level in the trend. The opinion in 2007 that increasing use of biofuels might lead to a decrease in soybean oil supply caused soymeal prices to rise, which indirectly made soybean prices rise. Skew levels also acted as psychological levels on this occasion. Soybean prices experienced roller-coaster-type fluctuations from 2007 to 2008, as shown in Figure 3. To stabilize soybean prices, the government implemented a temporary reserve policy for soybeans from 2008 to 2013, which formed strong support. Significant skew phenomena in 2009 and 2010 were partly attributable to government intervention. Skew levels may not only reflect tangible government intervention in the market, but also the effectiveness of policy through the value of skew probabilities. The more that skew probability deviates from 0.5 , the more effective government policy is. According to the Ministry of Agriculture, the ratio of government reserves to yields reached $20 \%$ for 2009 and 2010. Such strong government intervention policy is demonstrated by the high skew probability in 2010 . 
Table 4: Parameter estimation results of skew OU model in strong gluten wheat futures market

\begin{tabular}{|c|c|c|c|c|c|c|c|c|c|}
\hline \multirow{2}{*}{ Year } & \multicolumn{7}{|c|}{ Skew OU estimates } & \multicolumn{2}{|c|}{ OU estimates } \\
\hline & $\hat{\kappa}$ & $\hat{\theta}$ & $\hat{\sigma}$ & $\hat{a}$ & $\hat{p}$ & $p$-value ${ }^{1}$ & $p$-value ${ }^{2}$ & $\hat{\kappa}_{0}$ & $\hat{\sigma}_{0}$ \\
\hline \multirow{3}{*}{2004} & & & & 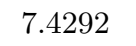 & & & & 1.0000 & \\
\hline & 46) & 2) & $.0054)$ & & $92)$ & & & 20) (0.0645) & 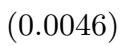 \\
\hline & {$[0.5170]$} & {$[0.9386]$} & {$[-0.3093]$} & {$[0.5715]$} & {$[0.5115]$} & {$[297.8248]$} & {$[288.6072]$} & {$[1047.5660]$} & \\
\hline \multirow{3}{*}{2005} & 1.0631 & 7.4336 & 0.0739 & 7.4158 & 0.3021 & 0.0012 & 0.0013 & 7.3669 & 0.0763 \\
\hline & $(0.5268)$ & $(0.0972)$ & $(0.0034)$ & $(0.0037)$ & $(0.1128)$ & $(124.6721)$ & $(114.6502)$ & $(2.9261) \quad(0.2559)$ & $(0.0036)$ \\
\hline & {$[1.0968]$} & {$[0.7515]$} & {$[0.3797]$} & {$[0.8538]$} & {$[-1.0259]$} & {$[299.0466]$} & {$[292.7448]$} & {$[1113.2548]$} & \\
\hline \multirow{3}{*}{2006} & & & & & & & & -0.2472 & 0.1173 \\
\hline & $(0.1248)$ & $(0.0876)$ & $(0.0063)$ & $(0.0291)$ & $(0.2648)$ & $(323.3226)$ & $(276.2116)$ & $(1.6025) \quad(0.5619)$ & $(0.0$ \\
\hline & {$[1.3278]$} & {$[0.4310]$} & {$[0.9666]$} & {$[0.3803]$} & {$[0.4165]$} & {$[398.5174]$} & {$[295.3736]$} & {$[1049.5686]$} & \\
\hline \multirow{3}{*}{2007} & & & & 7.5127 & & & & 7.5578 & \\
\hline & $(0.4254)$ & $(0.0247)$ & $(0.0061)$ & $(0.0459)$ & $(0.1703)$ & $(337$ & $(336.3998)$ & $(2.1850) \quad(0.1008)$ & $(0.0$ \\
\hline & {$[0.25$} & {$[-0.0918]$} & {$[1.2161]$} & {$[-0.0508]$} & {$[0.9953]$} & $972]$ & 844] & {$[1008$.} & \\
\hline \multirow{3}{*}{2008} & 1.4080 & & 0.1105 & 7.5907 & 0.6494 & & & 7.5747 & \\
\hline & $(0.61$ & & & & & & & $310) \quad(0.0$ & \\
\hline & {$[0.7$} & {$[-0.2040]$} & {$[-0.7687]$} & {$[0.9581]$} & {$[-0.4843]$} & {$[403$} & {$[402$} & {$[1041.8777]$} & \\
\hline \multirow{3}{*}{2009} & 1.9695 & 7.6516 & 0.0516 & 7.6526 & 0.7458 & $<0$ & 0.0002 & 7.6422 & 0.0 \\
\hline & $(1.0422)$ & 7) & $(0$. & 096) & & & & $(2.0982) \quad(0.0$ & \\
\hline & {$[0.7784]$} & {$[0.9094]$} & {$[-0.1568]$} & {$[1.5949]$} & {$[-0.5734]$} & 024] & {$[401$.} & [1231.6995] & \\
\hline \multirow{3}{*}{2010} & 0.5 & & & 7.8914 & & & & 0.2857 & 0.0 \\
\hline & $(0.2454)$ & $(0.0837)$ & $(0.0033)$ & $(0.0033)$ & $(0$. & 1) & 4) & $(0.9467) \quad(1.1\}$ & $(0.0$ \\
\hline & {$[-0.8237]$} & {$[-0.5471]$} & {$[-0.3294]$} & {$[-1.4264]$} & {$[-0.5413]$} & 740] & {$[299$} & {$[1163.7674]$} & \\
\hline \multirow{3}{*}{2011} & & & & 7.8616 & & & & 7.5559 & \\
\hline & $(0.7215)$ & $(0.1015)$ & $(0.0047)$ & $(0.0685)$ & $(0.2396)$ & $(39$ & & $(1.3561) \quad(0.59$ & \\
\hline & & & & & & & & {$[1102.5$} & \\
\hline \multirow{3}{*}{2012} & & & & & & & & 7.7587 & \\
\hline & $(0.81$ & $(0.0$ & $(0.0$ & 52) & 0) & $(58$ & & 89) (0.1000) & \\
\hline & {$[0.82$} & {$[1.4$} & {$[0.1$} & & & & & {$[1130$} & \\
\hline \multirow{3}{*}{2013} & 0.2793 & & & 7.9143 & 0.5095 & & & 7.8370 & \\
\hline & $(0.2607)$ & 833) & $(0$ & $(0.0327)$ & & $(327$ & & $(1.0787) \quad(0.1570)$ & \\
\hline & {$[-0.0699]$} & {$[0.1865]$} & {$[0.6519]$} & {$[1.0763]$} & {$[-0.9307]$} & {$[390.4920]$} & {$[384.3877]$} & [1203.2664] & \\
\hline \multirow{3}{*}{2014} & 0.7238 & & 0.0503 & 7.9525 & 0.3982 & 0.0003 & 0.0024 & 7.9572 & \\
\hline & $(0.4$ & & & & & & & $(1.4204) \quad(0$ & \\
\hline & {$[-0.3625]$} & {$[0.13$} & {$[0.2587]$} & {$[-0.0529]$} & {$[-1.3065]$} & {$[401.2676]$} & {$[376.1997]$} & [1244.9249] & \\
\hline \multirow{3}{*}{2015} & 0.3924 & & & 7.8461 & 0.6287 & $<0.0001$ & م 0010 & 7.8872 & 0.0 \\
\hline & $(0.3928)$ & $(0.0654)$ & $(0.0053)$ & $(0.0078)$ & $(0$. & 29) & 30) & $(1.7441) \quad(0.0834)$ & $(0.0029)$ \\
\hline & {$[0.0706]$} & {$[-0.0618]$} & {$[0.4621]$} & {$[0.3125]$} & {$[-0.5636]$} & {$[400.1120]$} & [390.6825] & [1196.2753] & \\
\hline \multirow{4}{*}{2016} & & & & & & & & 7.9772 & 0. \\
\hline & $(0.3291)$ & $(0.0059)$ & $(0.0036)$ & $(0.0363)$ & $(0.1566)$ & $(352.8038)$ & 789) & $(1.7191) \quad(0.0449)$ & $(0.0037)$ \\
\hline & {$[0.6241]$} & {$[0.1155]$} & {$[1.3970]$} & {$[-0.8408]$} & {$[0.9196]$} & {$[402.2591]$} & [400.9691] & [1107.1515] & \\
\hline & 0.1144 & 7.9221 & 0.0726 & 8.0209 & 0.2189 & $<0.0001$ & $<0.0001$ & -0.3403 & 0.0856 \\
\hline \multirow[t]{3}{*}{2017} & $(0.1071)$ & $(0.0804)$ & $(0.0037)$ & $(0.0079)$ & $(0.0498)$ & $(1669.2981)$ & $(1296.5001)$ & $(1.1652) \quad(0.2854)$ & $(0.0039)$ \\
\hline & {$[-0.6788]$} & {$[-1.2006]$} & & {$[0.0897]$} & & {$[396.3924]$} & & [1124.5755] & \\
\hline & 5.3994 & 7.8409 & 0.0658 & 7.8382 & 0.5708 & 0.0988 & 0.1459 & 7.8286 & 0.0684 \\
\hline \multirow[t]{2}{*}{2018} & $(1.1064)$ & $(0.0051)$ & $(0.0029)$ & $(0.0008)$ & $(0.0242)$ & $(391.8279)$ & $(372.6791)$ & $(4.5804) \quad(0.0418)$ & $(0.0033)$ \\
\hline & {$[-1.5714]$} & {$[-0.5880]$} & {$[1.2566]$} & {$[0.0360]$} & {$[-0.2934]$} & {$[391.5100]$} & [389.7579] & [1079.0134] & \\
\hline
\end{tabular}

Notes: Columns (2) to (6) report estimation results based on skew OU model via Bayesian methods. Standard errors are reported in parentheses. CD statistics are reported in square brackets. Column (7) reports $p$-value ${ }^{1}$ for whether the estimator of the skew probability is significantly different from 0.5 . Column (8) reports $p$ value $^{2}$ of the generalized quasi-likelihood ratio test for the null hypothesis that the OU model is more suitable than the skew OU model. Corresponding test statistics are reported in parentheses. To save space, the critical values at the significance level of $10 \%$ used are reported in square brackets. Columns (9) to (11) report estimation results based on the OU model via MLE methods. Standard errors are reported in parentheses. Values of the maximized log-likelihood function are reported in square brackets. The estimation period is based on daily observations from January 1, 2004 to December 22, 2018. 


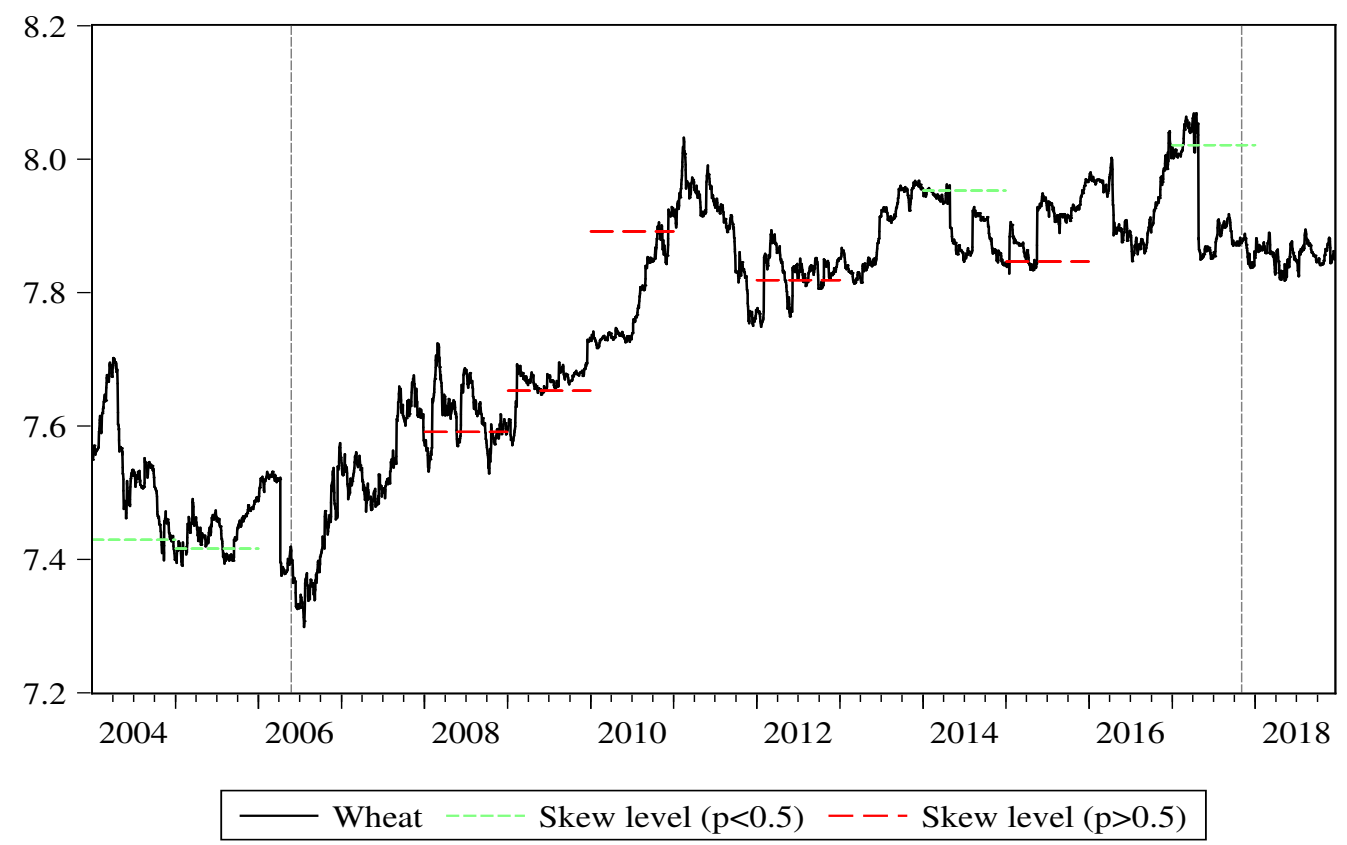

Figure 8: Skew phenomena in strong gluten wheat futures market

Notes: This figure shows logarithmic prices of strong gluten wheat futures (solid curve) with skew levels where skew probabilities are significantly larger than 0.5 (horizontal red dashed lines) and skew levels where skew probabilities are significantly smaller than 0.5 (horizontal green dashed lines). The vertical dashed lines illustrate policy-change split points. The first one marks the government for the first time implementing the floor purchase price policy for wheat on May 19, 2006. The second one shows that the NDRC cut the minimum purchase price for wheat for the first time for 12 years on October 27, 2017, which was negative market news. The sample period extends from January 1, 2004 to December 22, 2018.

Since implementation of the temporary reserve policy, prices of domestic soybean became much higher than imported soybean. The ratio of government reserves to yields was less than $10 \%$ for 2012 and 2013. Therefore, the effectiveness of government intervention was weakened in this case. The temporary reserve policy for soybean was stopped in 2014 and the government started trials of a target price subsidy policy in the same year. Soybean prices began to be determined more by the market, and government no longer directly intervened in market prices. When the average market price was lower than the target price for a certain period, the state would subsidize price differences to farmers. When the market price was high, government did not grant subsidies. This change could be regarded as the start of policy reform. This could partly explain why there were no significant skew phenomena for six years from 2010. The year 2017 showed a strong pressure level as prices were at relatively high levels and skew probability was less than 0.5. The destocking of soybean caused the market to have a higher probability of decline. In the same year, the government abandoned the target price subsidy policy and changed to a direct producer subsidy policy.

The CD statistics in Table 5 suggest that convergence is attained for Markov chains. According to the results of a generalized quasi-likelihood ratio test, the skew OU model performs significantly better than the OU model at the $10 \%$ significance level in the No. 1 soybean market when skew phenomena are significant. In addition, we report the estimation results of MLE for the OU model, with parameters $\kappa, \theta$ and $\sigma$. Parameters $\kappa$ in the OU model are not significant in most years. These 
results show that the skew OU model is superior to the OU model when modeling the Chinese No. 1 soybean futures market with government controls.

Table 5: Parameter estimation results of skew OU model in No. 1 soybean futures market

\begin{tabular}{|c|c|c|c|c|c|c|c|c|c|c|}
\hline \multirow{2}{*}{ Year } & \multicolumn{7}{|c|}{ Skew OU estimates } & \multicolumn{3}{|c|}{ OU estimates } \\
\hline & $\hat{\kappa}$ & $\hat{\theta}$ & $\hat{\sigma}$ & $\hat{a}$ & $\hat{p}$ & $p$-value ${ }^{1}$ & $p$-value ${ }^{2}$ & $\hat{\kappa}_{0}$ & $\hat{\theta}_{0}$ & $\hat{\sigma}_{0}$ \\
\hline \multirow{3}{*}{2003} & 1.4783 & 53 & 0.1381 & 64 & 0.2840 & 1 & 7 & 0.8833 & 8.3510 & 0.1397 \\
\hline & $(0.7009)$ & $(0.0959)$ & $(0.0068)$ & $.0942)$ & $(0.3044)$ & $(296.8853)$ & $(287.8277)$ & $(1.4277)$ & $(0.7079)$ & $(0.0065)$ \\
\hline & {$[-1.1189]$} & {$[0.5124]$} & {$[0.3215]$} & {$[1.5766]$} & {$[1.5642]$} & [299.9424] & {$[300.5365]$} & & {$[974.0207]$} & \\
\hline \multirow{3}{*}{2004} & 0.4146 & 8.0900 & & & 0.1646 & & 0.0006 & 0.0384 & 7.0114 & 0.1729 \\
\hline & $(0.1845)$ & $(0.0704)$ & $(0.0077)$ & $(0.0217)$ & $(0.0731)$ & $(720.7043)$ & $(645.3221)$ & $(1.1786)$ & $(31.7260)$ & $(0.0079)$ \\
\hline & {$[0.9456]$} & {$[-0.4225]$} & {$[1.2279]$} & {$[-0.1391]$} & {$[0.4759]$} & {$[398.4501]$} & {$[396.7737]$} & & {$[965.3000]$} & \\
\hline \multirow{3}{*}{2005} & 5.9215 & 7.9357 & 0.1356 & 8.0036 & 0.3963 & 0.3394 & 0.3672 & 5.2200 & 7.9359 & 0.1388 \\
\hline & $(1.0294)$ & $(0.0068)$ & $(0.0067)$ & $(0.0483)$ & $(0.1536)$ & $(356.0$ & $(348$ & $(2.1345)$ & $(0.0286)$ & $(0.0065)$ \\
\hline & {$[-0.0364]$} & {$[0.7594]$} & {$[0.0975]$} & {$[0.8535]$} & {$[0.1657]$} & [411.5966] & 624] & & [971.3505] & \\
\hline \multirow{3}{*}{2006} & 2.4382 & 7.8063 & 0.0960 & 7.8077 & 0.3965 & 0.0 & 0.0 & 2.3250 & 7.8062 & 0.1000 \\
\hline & $(0.3480)$ & $(0.0265)$ & $(0.0042)$ & $(0.0346)$ & $(0.2327)$ & $(21$ & $(18$ & $(2.1465)$ & $(0.0819)$ & $(0.0046)$ \\
\hline & {$[1.0509]$} & [-1.1959] & {$[-1.3833]$} & {$[-1.5721]$} & {$[0.4864]$} & [300.5590] & [294.9058] & & [1069.1474] & \\
\hline \multirow{3}{*}{2007} & 1.2242 & 8.2268 & 0.1301 & 8.0021 & 0.6400 & 0.0093 & & -1.7994 & 7.9293 & 0.1314 \\
\hline & $(1.0819)$ & $(0.0936)$ & $(0.0075)$ & $(0.0398)$ & $(0.1818)$ & $(48$ & $(476$ & $(0.9636)$ & $(0.1517)$ & $(0.0061)$ \\
\hline & [0.2069] & {$[-0.9910]$} & {$[-0.1770]$} & {$[1.6296]$} & {$[-1.3260]$} & {$[397$} & [372.9233] & & [992.4909] & \\
\hline \multirow{3}{*}{2008} & & & & & & & & & 8.3 & 0.2711 \\
\hline & $(0.1845)$ & $(0.0704)$ & $(0.0077)$ & $(0.0217)$ & $(0.0731)$ & $(336$. & $(330$. & $(1.7661)$ & $(0.1570)$ & $(0.0126)$ \\
\hline & {$[-0.00$} & & {$[1.6372]$} & {$[-0.7372]$} & {$[-0.4900]$} & {$[405$.} & {$[404.8$} & & {$[835.8767]$} & \\
\hline \multirow{3}{*}{2009} & 6.6383 & & & & & & & 5.5914 & 8.2 & 0.1474 \\
\hline & $(0.5376)$ & $(0.0030)$ & $(0.0063)$ & $(0.0294)$ & $(0.2138)$ & $(297$ & $(28$ & $(3.2485)$ & $(0.0413)$ & $(0.0069)$ \\
\hline & {$[-1.2765]$} & {$[1.4416]$} & {$[0.2167]$} & {$[0.9925]$} & {$[-0.5299]$} & {$[310.3224]$} & [307.5528] & & {$[965.9856]$} & \\
\hline \multirow{3}{*}{2010} & 0.9382 & 8.3228 & 0.1079 & 8.3279 & 0.7035 & 0.0515 & 0.0622 & 0.9328 & & 0.1069 \\
\hline & & & & & & & & 41) & & $(0.0050)$ \\
\hline & {$[0.9530]$} & [-0.8491] & {$[0.8743]$} & {$[0.7648]$} & [1.1832] & {$[297.5474]$} & [297.1545] & & [1017.6968] & \\
\hline \multirow{3}{*}{2011} & 9.0765 & 8.4089 & 0.0941 & 8.4217 & 0.4657 & 0.4598 & 0.4564 & 8.1027 & 8.4096 & 45 \\
\hline & $(0.8647)$ & $(0.0025)$ & $(0.0046)$ & $(0.0275)$ & $(0.1310)$ & $(34$ & $(344$ & $(3.3016)$ & $(0.0123)$ & $(0.0044)$ \\
\hline & {$[1.0523]$} & [-1.4095] & {$[-0.0557]$} & {$[-0.3322]$} & {$[-0.1885]$} & {$[419.2613]$} & [414.1819] & & [1054.6846] & \\
\hline \multirow{3}{*}{2012} & 3.7632 & & 0.0904 & & & & 0.4 & 3.3857 & 8.4494 & 0.0918 \\
\hline & $(0.4970)$ & $(0.0053)$ & $(0.0043)$ & $(0.0552)$ & $(0.1662)$ & $(341.9156)$ & $(334.9296)$ & $(2.2863)$ & $(0.0298)$ & $(0.0044)$ \\
\hline & {$[0.1073]$} & {$[-0.4901]$} & {$[-0.0468]$} & {$[-0.2103]$} & {$[-0.6909]$} & {$[408.7132]$} & {$[401.6794]$} & & [1042.7410] & \\
\hline \multirow{3}{*}{2013} & 2.2892 & 8.4547 & 0.0584 & 8.4401 & 0.3495 & 0.2744 & 0.3136 & 2.8193 & 8.4503 & 0.0609 \\
\hline & $(0.2964)$ & $(0.0039)$ & $(0.0027)$ & $(0.0186)$ & $(0.2443)$ & $(350.7903)$ & $(320.8941)$ & $(2.0206)$ & $(0.0235)$ & $(0.0029)$ \\
\hline & {$[-0.7471]$} & & {$[0.7741]$} & [0.1529] & [1.4726] & [392.3907] & [391.5739] & & $1139.5673]$ & \\
\hline \multirow{3}{*}{2014} & 10.6777 & 8.4105 & 0.0897 & 8.3902 & 0.2292 & 0.3766 & 0.3927 & 10.9440 & 8.4145 & 0.0907 \\
\hline & $(0.7567)$ & $(0.0016)$ & $(0.0046)$ & $(0.0081)$ & $(0.1200)$ & $(360.8008)$ & $(357.5476)$ & $(3.5870)$ & $(0.0101)$ & $(0.0042)$ \\
\hline & {$[0.4745]$} & {$[0.2966]$} & {$[0.7097]$} & {$[0.6425]$} & {$[-0.2089]$} & {$[430.0501]$} & {$[427.9445]$} & & [1106.1802] & \\
\hline \multirow{3}{*}{2015} & 1.8715 & 8.3242 & 0.1007 & 8.3333 & 0.5404 & 0.3747 & 0.4184 & 1.4627 & 8.1259 & 0.1000 \\
\hline & $(0.7517)$ & $(0.0916)$ & $(0.0058)$ & $(0.0237)$ & $(0.1192)$ & $(349.9926)$ & $(347.5607)$ & $(1.8057)$ & $(0.2587)$ & $(0.0047)$ \\
\hline & {$[-0.4669]$} & {$[-0.8793]$} & {$[0.3261]$} & {$[-1.3564]$} & {$[-0.5578]$} & {$[407.3435]$} & {$[415.2278]$} & & [1060.0387] & \\
\hline
\end{tabular}

The speed of mean reversion shows great variation in soymeal markets. The mean-reversion speed of soymeal in 2006 was greater than 10, indicating that, from the starting point to the long-term mean, the process took less time. Other parameters are similar in magnitude. Soymeal is mainly squeezed from imported transgenic soybeans and therefore more affected by international soybean prices. For soymeal futures, skew phenomena were present only in several years. Prices of soymeal are affected mainly by international soybean prices and are thus less influenced by government intervention. The skew probability was 0.2183 for 2002, 0.3745 for 2011, 0.2563 for 2012 and 0.3193 for 2017, showing relatively significant pressure levels. Soymeal was seriously overstocked in 2002. In 2011, many 
Table 5 (Continued)

\begin{tabular}{|c|c|c|c|c|c|c|c|c|c|c|}
\hline \multirow{2}{*}{ Year } & \multicolumn{7}{|c|}{ Skew OU estimates } & \multicolumn{3}{|c|}{ OU estimates } \\
\hline & $\hat{\kappa}$ & $\hat{\theta}$ & $\hat{\sigma}$ & $\hat{a}$ & $\hat{p}$ & $p$-value ${ }^{1}$ & $p$-value ${ }^{2}$ & $\hat{\kappa}_{0}$ & $\hat{\theta}_{0}$ & $\hat{\sigma}_{0}$ \\
\hline \multirow{4}{*}{2016} & 2 & 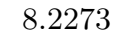 & 421 & 8.19 & 0.4149 & 581 & 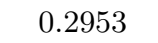 & 2.7486 & 8.2317 & 0.1418 \\
\hline & $(0.7530)$ & $(0.0121)$ & $(0.0099)$ & $(0.0779)$ & $(0.2035)$ & $(328.1071)$ & $(328.5458)$ & $(2.6107)$ & $(0.0553)$ & $(0.0066)$ \\
\hline & {$[-0.4037]$} & {$[-0.5122]$} & {$[0.4513]$} & {$[-0.1824]$} & {$[-0.8095]$} & [404.1758] & {$[402.2353]$} & & [974.8278] & \\
\hline & 1.2896 & 8.2344 & 0.0885 & 8.2801 & 0.3562 & 0.0428 & 0.0486 & 1.3661 & 8.1721 & 0.0882 \\
\hline \multirow[t]{3}{*}{2017} & $(0.4578)$ & $(0.0980)$ & $(0.0039)$ & $(0.0616)$ & $(0.1653)$ & $(442.2895)$ & $(442.0724)$ & $(1.9563)$ & $(0.1400)$ & $(0.0042)$ \\
\hline & {$[-0.4969]$} & {$[0.6677]$} & {$[-0.6600]$} & {$[-1.0359]$} & {$[0.1252]$} & [404.3415] & {$[407.5605]$} & & {$[1065.7271$} & \\
\hline & 2.8586 & 8.1943 & 0.1034 & 8.2322 & 0.3592 & 0.1180 & 0.0576 & 0.6130 & 7.6569 & 0.1025 \\
\hline \multirow[t]{2}{*}{2018} & $(0.9681)$ & $(0.1173)$ & $(0.0079)$ & $(0.0034)$ & $(0.0465)$ & $(409.3383)$ & $(411.8101)$ & $(2.4933)$ & $(2.2109)$ & $(0.0049)$ \\
\hline & [1.1204] & [0.0309] & [1.0144] & {$[-1.3695]$} & {$[-0.1840]$} & [415.6813] & [388.1889] & \multicolumn{3}{|c|}{ [1018.0001] } \\
\hline
\end{tabular}

Notes: Columns (2) to (6) report estimation results based on skew OU model via Bayesian methods. Standard errors are reported in parentheses. CD statistics are reported in square brackets. Column (7) reports $p$-value ${ }^{1}$ for whether the estimator of the skew probability is significantly different from 0.5. Column (8) reports $p$ value $^{2}$ of the generalized quasi-likelihood ratio test for the null hypothesis that the OU model is more suitable than the skew OU model. Corresponding test statistics are reported in parentheses. To save space, the critical values at the significance level of $10 \%$ used are reported in square brackets. Columns (9) to (11) report estimation results based on the OU model via MLE methods. Standard errors are reported in parentheses. Values of the maximized log-likelihood function are reported in square brackets. The estimation period is based on daily observations from January 1, 2003 to December 22, 2018.

factors, like the European Debt Crisis, high excess stock, and the nuclear crisis in Japan put pressure on prices. In 2012, high yield expectations in South America also put pressure on price dynamics. In addition, since late May, to curb the rise in soybean prices, the government increased auctions of reserve soybean. This policy played a macro-regulation role in the soymeal market. In 2017, negative factors like high yields depressed prices. Given the above, stock-to-use ratio, natural disasters, and other factors would also affect the price trend. Farmers and investors had different expectations about the impact of the same given event. Skew levels could reflect the imbalance between bullishness and bearishness. We show skew levels in Figure 10.

The CD statistics in Table 6 show that the Markov chains converge. According to the results of a generalized quasi-likelihood ratio test, the skew OU model performs significantly better than the OU model at the $10 \%$ significance level in the soymeal market when skew phenomena are significant. Additionally, we report the estimation results of MLE for the OU model, with parameters $\kappa, \theta$, and $\sigma$. Parameters $\kappa$ in the OU model are not significant in most cases. These results show that the skew OU model is superior to the OU model when modeling the Chinese soymeal futures market.

\subsection{Soft Futures Market}

Table 7 shows parameter estimation results from applying the skew OU model to cotton futures. The mean-reversion coefficient of cotton futures in 2017 was significantly greater than in other years. In cotton futures markets, years 2007, 2009, 2017, and 2018 showed significant support levels. There existed potential pressure levels in 2010, 2012, 2013, and 2014. Most skew probabilities in cotton futures markets were far from 0.5 , giving a first indication that corresponding skew levels were very strong. 


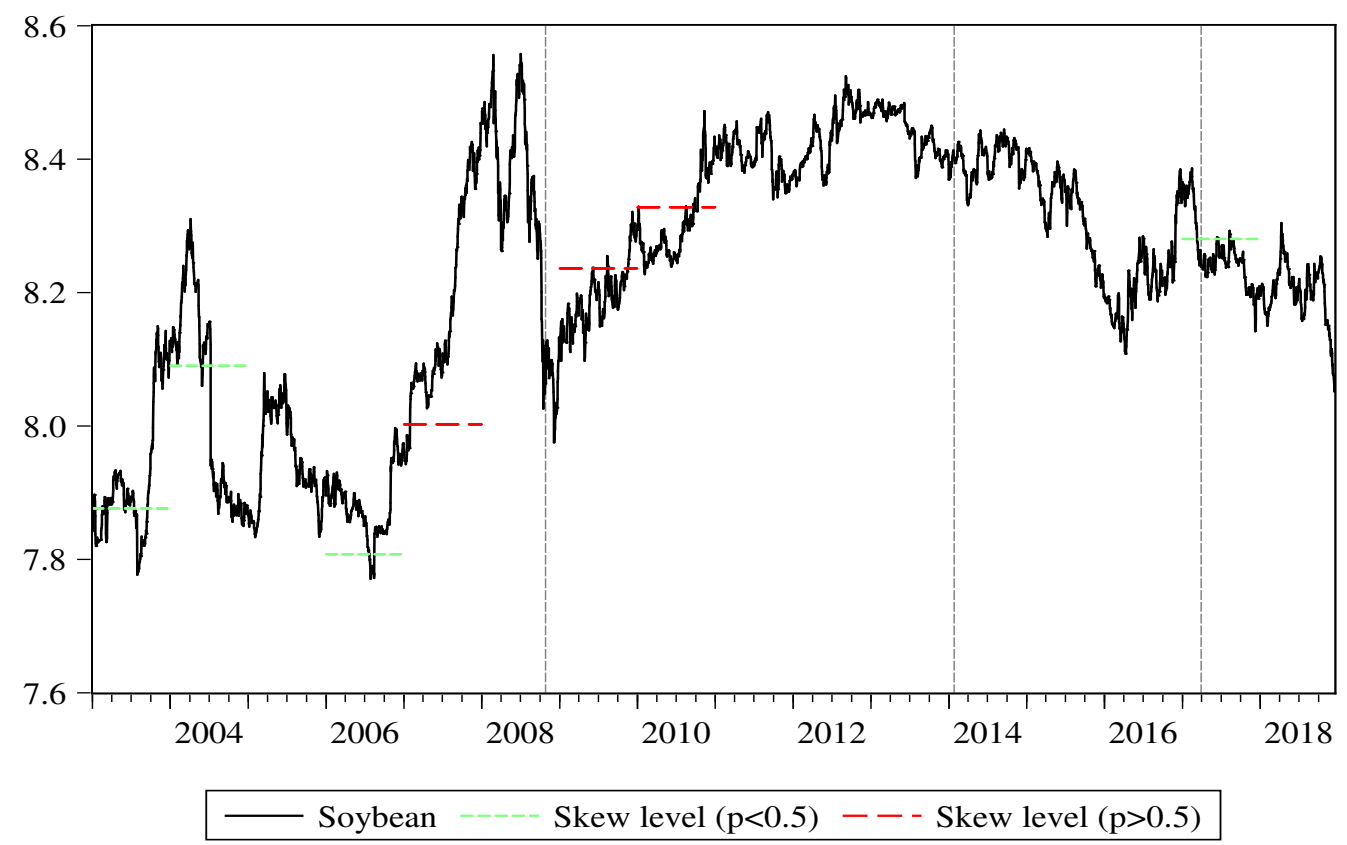

Figure 9: Skew phenomena in No. 1 soybean futures market

Notes: This figure shows logarithmic prices of No. 1 soybean futures (solid curve) with skew levels where skew probabilities are significantly greater than 0.5 (horizontal red dashed lines) and skew level where skew probabilities are significantly smaller than 0.5 (horizontal green dashed lines). The vertical dashed lines illustrate policy-change split points. The first one marks the government implementing the temporary reserve policy for soybeans from October 20, 2008, which formed strong support. The second one shows that such policy was stopped on January 19, 2014, and the government started trials of the target price subsidy policy in the same year. The last one indicates that on March 23, 2017, the government abandoned the target price subsidy policy and changed to a direct producer subsidy policy. The sample period extends from January 1 , 2003 to December 22, 2018.

The financial crisis of 2008 caused prices of agricultural commodities such as soybean and cotton to plummet. From August 21, 2008 to April 10, 2009, China purchased and stored 2.78 million tons of cotton. For the next two years, the NDRC suspended the policy to launch a cotton temporary state reserve program. However, cotton prices experienced a roller-coaster ride from 2010 to 2011, as shown in Figure 5. To protect the interests of farmers, the NDRC launched a cotton temporary state reserve program again in the spring of 2011 (March 2011). The policy established a purchase price of 19,800 RMB per ton, 35\% above the world cotton price. On March 2, 2012, the NDRC announced a new state reserve purchase price of 20,400 RMB per ton, $65 \%$ above the world price. This purchase price was maintained by the NDRC for 2013. The policy strongly supported prices for nearly three years and kept prices at a relatively high and stable level. As the domestic price was much higher than the cost of imports, cotton textile enterprises purchased raw materials from abroad and pressure on the government reserve increased sharply. According to the Ministry of Agriculture, the ratio of government reserves to production exceeded $90 \%$ in 2013. It would be interesting to observe how skew levels behaved as pressure levels in this case. From Figure 11, we observe that prices did not rise in these years. Thus, the skew OU model may reflect the true trend of market. There was potential downward pressure, although government policy maintained the price dynamics. Skew levels in Figure 
Table 6: Parameter estimation results of skew OU model in soymeal futures market

\begin{tabular}{|c|c|c|c|c|c|c|c|c|c|c|}
\hline \multirow{2}{*}{ Year } & \multicolumn{7}{|c|}{ Skew OU estimates } & \multicolumn{3}{|c|}{ OU estimates } \\
\hline & $\hat{\kappa}$ & $\hat{\theta}$ & $\hat{\sigma}$ & $\hat{a}$ & $\hat{p}$ & $p$-value ${ }^{1}$ & $p$-value ${ }^{2}$ & $\hat{\kappa}_{0}$ & $\hat{\theta}_{0}$ & $\hat{\sigma}_{0}$ \\
\hline \multirow{3}{*}{2001} & & & & & & & & $x^{-1}+2$ & 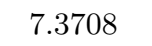 & \\
\hline & & & & & & & $(411.00$ & & $(0.0407)$ & \\
\hline & {$[-1.4085]$} & {$[0.9794]$} & {$[-0.1562]$} & {$[-0.9915]$} & {$[-0.6867]$} & {$[492.9921]$} & {$[467.8205]$} & & [1028.2475] & \\
\hline \multirow{3}{*}{2002} & 0.5268 & 7.4772 & 0.0785 & 7.3907 & 0.4387 & 0.0259 & 0.0158 & 0.1185 & 8.0790 & 0.0001 \\
\hline & $(0.2795)$ & $(0.0980)$ & $(0.0037)$ & $.0165)$ & $(0.2183)$ & $(260.2259)$ & $(245.2872)$ & $(1.3948)$ & $(7.2437)$ & $(0.0038)$ \\
\hline & {$[0.3809]$} & {$[-0.2886]$} & {$[0.3424]$} & {$[0.7948]$} & {$[0.6897]$} & {$[299.1455]$} & [296.5196] & & [1082.7358] & \\
\hline \multirow{3}{*}{2003} & 0.9903 & & 0.1406 & 7.6656 & 0.5369 & 0.2199 & 0.1765 & 0.7739 & 8.1120 & 0.1407 \\
\hline & $(0.5117)$ & $(0.1123)$ & $(0.0069)$ & $(0.0954)$ & $(0.1803)$ & $(320.1025)$ & $(314.1956)$ & $(1.1488)$ & $(0.6386)$ & $(0.0066)$ \\
\hline & {$[0.5410]$} & {$[-0.4112]$} & {$[0.8543]$} & {$[1.3729]$} & {$[-0.3868]$} & {$[395.5619]$} & {$[38$} & & {$[955.5229]$} & \\
\hline \multirow{3}{*}{2004} & & & & & & & & & & 0.2035 \\
\hline & $(0.1$ & $(0$. & 097) & $(0$ & $(0.2265)$ & 769) & $(32 \tau$ & $(1.3600)$ & $(1.0228)$ & $(0.0094)$ \\
\hline & {$[-0.3294]$} & {$[-0.5226]$} & {$[-0.9604]$} & & {$[-0.4153]$} & {$[397.4772]$} & [399.5967] & & [914.5757] & \\
\hline \multirow{3}{*}{2005} & & & & & & 0.9295 & & & & \\
\hline & $(0.5$ & $(0$. & & & & $(31$ & $(29$ & $(2.0424)$ & & $(0.0072)$ \\
\hline & {$[-0$.} & {$[-0$.} & {$[-0.7629]$} & {$[-1.2401]$} & {$[-0.1614]$} & 1430] & {$[30$} & & {$[932$.} & \\
\hline \multirow{3}{*}{2006} & & & & & & & & & & 0 . \\
\hline & & & & & & & & $(4.1997)$ & & \\
\hline & {$[0.0654]$} & {$[-1.2583]$} & {$[1.3697]$} & {$[0.1153]$} & {$[-0.0987]$} & [439.8630] & {$[427.6741]$} & & {$[1015$.} & \\
\hline \multirow{3}{*}{2007} & & 7.9739 & 0.1491 & & 0.4594 & 0.3938 & 0.1216 & -2.2844 & 7.79 & 0.1470 \\
\hline & $(0.9$ & 55) & 9) & & & 200) & $(35$ & $(1.3181)$ & 8) & $69)$ \\
\hline & {$[-0.0580]$} & {$[-0.6190]$} & {$[-0.6764]$} & [1.2218] & {$[-0.3134]$} & {$[396.7047]$} & 2309] & & [954.0166] & \\
\hline \multirow{3}{*}{2008} & & & & & & & & & & \\
\hline & $(0.4121)$ & $(0.0804)$ & $(0.0143)$ & $(0.09$ & $(0.2618)$ & 7010) & $(28$ & $(1.6690)$ & $(0.2478)$ & $(0.0130)$ \\
\hline & [1.0489] & {$[-0.1882]$} & {$[1.3300]$} & {$[-1.4421]$} & {$[-0.5260]$} & {$[402.6970]$} & [288.2498] & & [819.6612] & \\
\hline \multirow{3}{*}{2009} & & & & & & & & 82 & 8.00 & 10 \\
\hline & $(0.4$ & 3) & 8) & 0) & $(0$. & 323) & $(41$ & 88) & $(0.0$ & $(0$. \\
\hline & & & & & & & & & & \\
\hline \multirow{3}{*}{2010} & & & & & & & & & 8.3771 & 0.1206 \\
\hline & $(0.4$ & 889) & 7) & $(0$. & & 995) & $(32$ & $(1.7874)$ & $(2.1237)$ & $(0.0056)$ \\
\hline & {$[1.0$} & {$[0.5101]$} & {$[0.5580]$} & {$[0.9525]$} & {$[0.3405]$} & [397.1365] & [392.5100] & & [1012.1874] & \\
\hline \multirow{3}{*}{2011} & 1.3773 & 8.0472 & & 8.0145 & 0.3745 & 0.0951 & 0.0919 & (2) & 8.0445 & 0.10 \\
\hline & & & & & & & & 193) & & \\
\hline & {$[-0.1$} & {$[1.4204]$} & {$[-1.3404]$} & {$[1.4820]$} & {$[-0.2369]$} & [295.9383] & {$[293.9356]$} & & [1020.8100] & \\
\hline \multirow{3}{*}{2012} & & & & & & & & & 8.4902 & \\
\hline & $(0.6320)$ & $(0.1030)$ & $(0.0067)$ & $(0.0745)$ & $(0.2211)$ & $(571.8305)$ & $(572.7913)$ & $(1.4553)$ & $(0.6396)$ & $(0.0070)$ \\
\hline & {$[-0.4014]$} & {$[0.3521]$} & {$[0.7714]$} & [1.6483] & {$[1.0766]$} & [399.0703] & [396.9783] & & [962.3314] & \\
\hline \multirow{3}{*}{2013} & & & & & & & & & & to \\
\hline & $(0.1848)$ & $(0.0096)$ & $(0.0063)$ & $(0.0055)$ & $(0.0488)$ & $(359.8151)$ & $(290.8086)$ & $(3.1519)$ & $(0.0438)$ & $(0.0068)$ \\
\hline & [1.6157] & {$[-0.0947]$} & {$[-1.6242]$} & {$[-1.3945]$} & {$[0.3717]$} & [396.4205] & [298.5343] & & [970.1129] & \\
\hline \multirow{3}{*}{2014} & 0.5461 & 8.0432 & 0.1252 & 8.0989 & 0.5274 & 0.3872 & 0.4611 & -1.0326 & 8.1658 & 0.1255 \\
\hline & $(0.5280)$ & $(0.0935)$ & $(0.0071)$ & $(0.1288)$ & $(0.2408)$ & $(336.9429)$ & $(337.3194)$ & $(1.4440)$ & $(0.1790)$ & $(0.0059)$ \\
\hline & {$[-0.1824]$} & {$[-0.3796]$} & {$[-0.6299]$} & & {$[-0.1929]$} & {$[400.2315]$} & [389.3944] & & [1003.0602] & \\
\hline \multirow{3}{*}{2015} & 1.9795 & 7.8726 & 0.1179 & 7.8682 & 0.4526 & 0.2201 & 0.2497 & 1.8672 & 7.7479 & 0.1157 \\
\hline & (1.0992) & $(0.0986)$ & $(0.0063)$ & $(0.0804)$ & $(0.1272)$ & $(321.6554)$ & $(325.0563)$ & $(2.1741)$ & $(0.1658)$ & $(0.0054)$ \\
\hline & {$[-0.9297]$} & {$[-1.6202]$} & {$[-0.2890]$} & {$[1.3687]$} & {$[1.1131]$} & [404.4687] & {$[414.2212]$} & & [1017.5159] & \\
\hline
\end{tabular}

11 backed up these phenomena in a more intuitive way. Similar phenomena could also be detected in corn markets. In addition, although China had imposed quotas on cotton imports, the volume of imports was several million tons higher than in previous years. The effectiveness of government intervention policy was impacted by international cotton prices. MacDonald et al. (2015) point out that China's introduction of a temporary cotton reserve program created a price floor, but this "soft" 
Table 6 (Continued)

\begin{tabular}{|c|c|c|c|c|c|c|c|c|c|c|}
\hline \multirow{2}{*}{ Year } & \multicolumn{7}{|c|}{ Skew OU estimates } & \multicolumn{3}{|c|}{ OU estimates } \\
\hline & $\hat{\kappa}$ & $\hat{\theta}$ & $\hat{\sigma}$ & $\hat{a}$ & $\hat{p}$ & $p$-value $^{1}$ & $p$-value ${ }^{2}$ & $\hat{\kappa}_{0}$ & $\hat{\theta}_{0}$ & $\hat{\sigma}_{0}$ \\
\hline \multirow{4}{*}{2016} & 1.3785 & 8.0456 & 583 & 7.8923 & 0.4522 & 910 & 1434 & 1.4000 & 8.0997 & 0.1624 \\
\hline & $(0.1555)$ & $(0.0082)$ & $(0.0087)$ & $(0.0164)$ & $(0.2116)$ & $(323.8808)$ & $(315.6145)$ & $(1.5875)$ & $(0.2320)$ & $(0.007$ \\
\hline & {$[0.4078]$} & {$[-0.7537]$} & {$[-0.1201]$} & {$[0.5628]$} & {$[0.1928]$} & [403.1212] & [401.9389] & & {$[951.7712]$} & \\
\hline & 5.7710 & 7.9411 & 0.0968 & 7.9258 & 0.3193 & 0.0528 & 0.0433 & 6.0167 & 7.9396 & 0.0989 \\
\hline \multirow[t]{2}{*}{2017} & $(0.6188)$ & $(0.0026)$ & $(0.0052)$ & $(0.0284)$ & $(0.2077)$ & $(279.1170)$ & $(273.6606)$ & $(3.7029)$ & $(0.0171)$ & $(0.0046)$ \\
\hline & {$[-0.1078]$} & {$[-1.3395]$} & {$[-0.6955]$} & {$[0.2607]$} & {$[-0.6101]$} & [302.3935] & {$[299.7747]$} & & {$[1067.2507]$} & \\
\hline \multirow{3}{*}{2018} & 4.3869 & 8.0172 & 0.1229 & 8.0239 & 0.2898 & 0.0311 & 0.1278 & 3.5042 & 8.0155 & 0.1387 \\
\hline & $(0.1763)$ & $(0.0038)$ & $(0.0062)$ & $(0.0017)$ & $(0.0153)$ & $(482.4528)$ & $(380.3779)$ & $(2.2649)$ & $(0.0425)$ & $(0.0065)$ \\
\hline & {$[-1.1655]$} & {$[-0.2541]$} & {$[-0.2416]$} & {$[0.8055]$} & {$[-0.2862]$} & {$[418.5567]$} & [390.2440] & & {$[958.7297]$} & \\
\hline
\end{tabular}

Notes: Columns (2) to (6) report estimation results based on the skew OU model via Bayesian methods. Standard errors are reported in parentheses. CD statistics are reported in square brackets. Column (7) reports $p$-value ${ }^{1}$ for whether the estimator of the skew probability is significantly different from 0.5. Column (8) reports $p$-value ${ }^{2}$ of the generalized quasi-likelihood ratio test for the null hypothesis that the OU model is more suitable than the skew OU model. Corresponding test statistics are reported in parentheses. To save space, the critical values at the significance level of $10 \%$ used are reported in square brackets. Columns (9) to (11) report estimation results based on the OU model via MLE methods. Standard errors are reported in parentheses. Values of the maximized log-likelihood function are reported in square brackets. The estimation period is based on daily observations from January 1, 2001 to December 22, 2018.

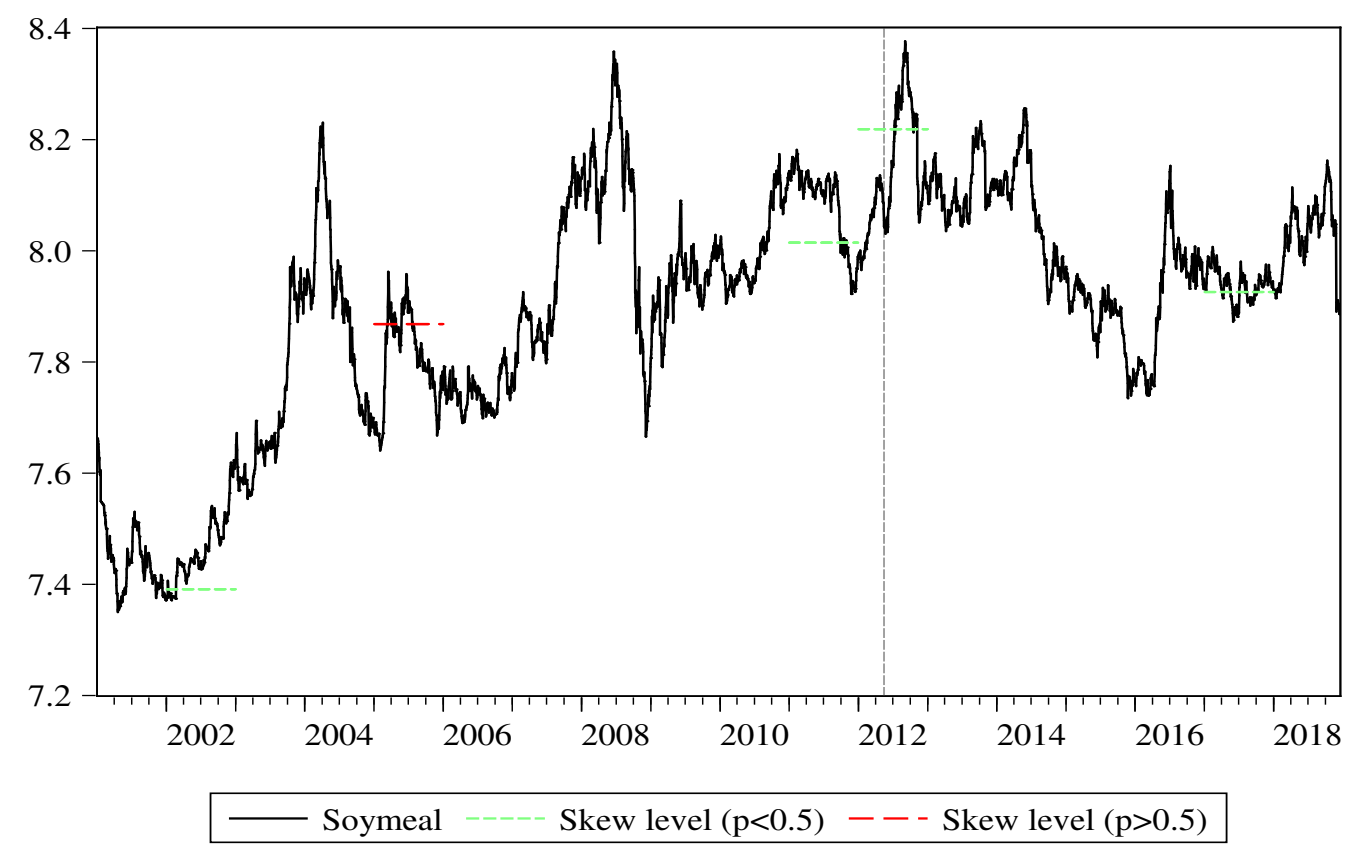

Figure 10: Skew phenomena in soymeal futures market

Notes: This figure shows logarithmic prices of soymeal futures (solid curve) with skew level, where skew probability is significantly greater than 0.5 (horizontal red dashed line) and skew levels where skew probabilities are significantly smaller than 0.5 (horizontal green dashed lines). The vertical dashed line illustrates the policy-change split point. It indicates that, since May 8, 2012, to curb the rise in soybean prices that year, the government increased auctions of reserve soybean. The sample period extends from January 1, 2001 to December 22, 2018.

price support program proved costly and unsustainable. 
To better influence the market in the formation of cotton prices, the government for the first time proposed that the temporary reserve program for cotton not be implemented in 2014. The government changed its policy toward target price subsidies in the same year. The stock-to-use ratio of cotton reached $154 \%^{13}$ and the pressure from destocking caused prices to fall. The skew probability in 2014 was far less than 0.5 , reflecting the change in government policy. We again attribute this significant phenomenon to government regulation. The target price of cotton had been steadily declining yearly to reflect the fair market value. It declined from 19,800 RMB per ton in 2014 to 19,100 RMB per ton in 2015 , and then to 18,600 RMB per ton in $2016^{14}$. Price differences between domestic and international cotton tended to narrow. On the basis of straightening out the price difference and destocking, government policy worked again. Significant skew phenomena in the final two years indicate this.

The CD statistics in Table 7 show that the Markov chains converge, which indicates the effectiveness of MCMC estimation. A generalized quasi-likelihood ratio test is statistically significant at the $10 \%$ level, indicating that the skew OU model is superior to the OU model when skew phenomena are significant in cotton futures markets. In addition, we report the estimation results of MLE for the OU model, with parameters $\kappa, \theta$ and $\sigma$. Parameters $\kappa$ in the OU model are not significant compared to those in the skew OU model. These results show that the skew OU model is superior to the OU model when modeling the Chinese cotton futures market with government regulation.

There are only four years with significant skew phenomena for white sugar futures. However, corresponding skew probabilities are all far from 0.5, meaning that skew levels are very strong. Imported sugar has a great influence on trends in sugar prices in China. Even with restricted quotas, the impact of sugar smuggling remains, in that it impacts the effectiveness of government intervention. Among the major sugar production countries of the world, China and Australia are the only two not to implement high tariff protections, which determines the strong correlation between domestic and international sugar prices. In the sugar market, there is also a temporary reserve policy. However, according to the Ministry of Agriculture, the white sugar reserve is relatively small. Therefore, the effectiveness of the policy is limited. As stated above, white sugar prices are more affected by growth habit. The years 2008, 2010, and 2017 showed strong price pressure levels, while 2016 showed a supporting level. Skew levels in softs markets are shown in Figure 12 in a more intuitive way. Because of the financial crisis of 2008 and the increase in yields, prices of white sugar futures had a large probability of going down. In 2010, the Chinese government implemented large-scale destocking, putting prices under pressure. Sugar yields fell more than expected in late 2015 and yields and the consumption gap significantly increased, which formed strong support for prices in the following year. White sugar started a new production cycle in 2017 and the high probability of falling prices is not surprising.

The CD statistics in Table 8 show that the Markov chains converge. A generalized quasi-likelihood ratio test is statistically significant at the $10 \%$ level, indicating that the skew OU model is superior to the OU model when skew phenomena are significant in white sugar futures markets. In addition, we report the estimation results of MLE for the OU model, with parameters $\kappa, \theta$, and $\sigma$. Parameters $\kappa$ in the OU model are not always significant. These results show that the skew OU model is superior to the OU model when modeling the Chinese white sugar futures market with regulation.

\footnotetext{
${ }^{13}$ Data are from USDA.

${ }^{14}$ Data are from NDRC.
} 
Table 7: Parameter estimation results of skew OU model in cotton futures market

\begin{tabular}{|c|c|c|c|c|c|c|c|c|c|c|}
\hline \multirow{2}{*}{ Yea } & \multicolumn{7}{|c|}{ Skew OU estimates } & \multicolumn{3}{|c|}{ OU estimates } \\
\hline & $\hat{\kappa}$ & $\bar{\theta}$ & $\hat{\sigma}$ & $\hat{a}$ & $\hat{p}$ & $p$-value $^{1}$ & $p$-value ${ }^{2}$ & $\hat{\kappa}_{0}$ & $\hat{\theta}_{0}$ & $\hat{\sigma}_{0}$ \\
\hline \multirow{3}{*}{2005} & & & & & & & & 66620 & & 1079 \\
\hline & & & & & & & & & & \\
\hline & {$[0.6229]$} & [1.3088] & {$[-0.8536]$} & $1.3923]$ & {$[0.6696]$} & {$[438$} & {$[43$} & & [1025.9676] & \\
\hline \multirow{3}{*}{2006} & & & 0.0704 & -27 & 0.6261 & & & 2.0002 & 9.5010 & \\
\hline & & & & & & & & & & \\
\hline & {$[1.1916]$} & {$[1.0604]$} & {$[-1.5705]$} & $1.4057]$ & {$[1.4452]$} & {$[425$} & {$[45$} & & [1127.6786] & \\
\hline \multirow{3}{*}{2007} & 0.63 & 3 & 0.0866 & 9.5 & 0.5641 & & & 0.3213 & 9.3 & 0 \\
\hline & & & & & & & & & & \\
\hline & {$[-0.9473]$} & {$[-0.5938]$} & {$[-0.1979]$} & {$[0.3$} & {$[1.4460]$} & {$[39$} & {$[39$} & & {$[1087$} & \\
\hline \multirow{3}{*}{2008} & & & & & & & & & & \\
\hline & $(0.4$ & 10 & 71) & 5) & 59) & & & $(1.3913)$ & $(0.0$ & \\
\hline & {$[-1.3$} & {$[-0$} & {$[-1$.} & & & {$[41$} & & & & \\
\hline \multirow{3}{*}{2009} & & & & & & & & & & \\
\hline & $(0.4$ & ( & 9) & 8) & $(0.2$ & $(28$ & & $948)$ & $(26$ & \\
\hline & {$[1.5$} & {$[-1$} & & & & & & & & \\
\hline \multirow{3}{*}{2010} & & & & & & & & & & \\
\hline & & & & & & & & & & \\
\hline & {$[-0$} & {$[0$} & {$[0$.} & & 79] & {$[30$} & & & & \\
\hline \multirow{3}{*}{2011} & & & & & & & & & & \\
\hline & & & & & & & & & & \\
\hline & {$[0$.} & {$[-0$.} & {$[0$.} & & {$[-0.4278]$} & {$[40$} & & & & \\
\hline \multirow{3}{*}{2012} & & & & & & & & & & \\
\hline & & & & & & & & & & \\
\hline & {$[-0.3$} & & {$[-0$.} & & {$[-0$.} & {$[40$} & & & {$[1131$} & \\
\hline \multirow{3}{*}{2013} & & & & & & & & & & \\
\hline & & & & & & & & & & \\
\hline & & & & & & & & & {$[1183$} & \\
\hline \multirow{3}{*}{2014} & & & & & & & & & & \\
\hline & & 然 & & & 52) & (14 & & & $(1)$ & \\
\hline & & & & & & & & & & \\
\hline & & & & & & & & & & \\
\hline \multirow[t]{2}{*}{2015} & & 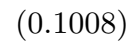 & & & & & & 1822) & $(0.4$ & \\
\hline & {$[-1$} & {$[1$.} & {$[-0.3694]$} & {$[-0$} & {$[-1.0206]$} & [400.1065] & $991]$ & & {$[1128$} & \\
\hline \multirow{3}{*}{2016} & 0 & & 0.1872 & & 0.5297 & & & & & \\
\hline & & & & & & & & & & \\
\hline & & {$[-0.3706]$} & {$[1.0382]$} & {$[-0.8583]$} & {$[-1.4400]$} & 440] & 541] & & {$[904$.} & \\
\hline \multirow{3}{*}{2017} & & & & & & & & & & \\
\hline & $(0$. & $(0.0025)$ & $(0$ & 0) & $(0.1664)$ & $(41$ & & $(4.5380)$ & $(0.0158)$ & $(0.004$ \\
\hline & [0.6699] & [0.2233] & {$[-1.6406]$} & {$[-1.1599]$} & {$[0.6627]$} & {$[411$} & 736] & & {$[1065$} & \\
\hline \multirow{3}{*}{2018} & & & & & & & & & & \\
\hline & $(0.8489)$ & $(0.1065)$ & $(0.0052)$ & $(0.0086)$ & $(0.1562)$ & $(597.7851)$ & $(608.7934)$ & $(1.8562)$ & $(0.0637)$ & $(0.004$ \\
\hline & {$[-1.3837]$} & {$[-0.2744]$} & {$[-0.0959]$} & {$[-0.2690]$} & {$[0.4131]$} & {$[385.5117]$} & {$[383.7912]$} & & [991.5493] & \\
\hline
\end{tabular}

Notes: Columns (2) to (6) report estimation results based on the skew OU model via Bayesian methods. Standard errors are reported in parentheses. CD statistics are reported in square brackets. Column (7) reports $p$-value ${ }^{1}$ for whether the estimator of the skew probability is significantly different from 0.5 . Column (8) reports $p$-value ${ }^{2}$ of the generalized quasi-likelihood ratio test for the null hypothesis that the OU model is more suitable than the skew OU model. Corresponding test statistics are reported in parentheses. To save space, the critical values at the significance level of $10 \%$ used are reported in square brackets. Columns (9) to (11) report estimation results based on the OU model via MLE methods. Standard errors are reported in parentheses. Values of the maximized log-likelihood function are reported in square brackets. The estimation period is based on daily observations from January 1, 2005 to December 22, 2018. 


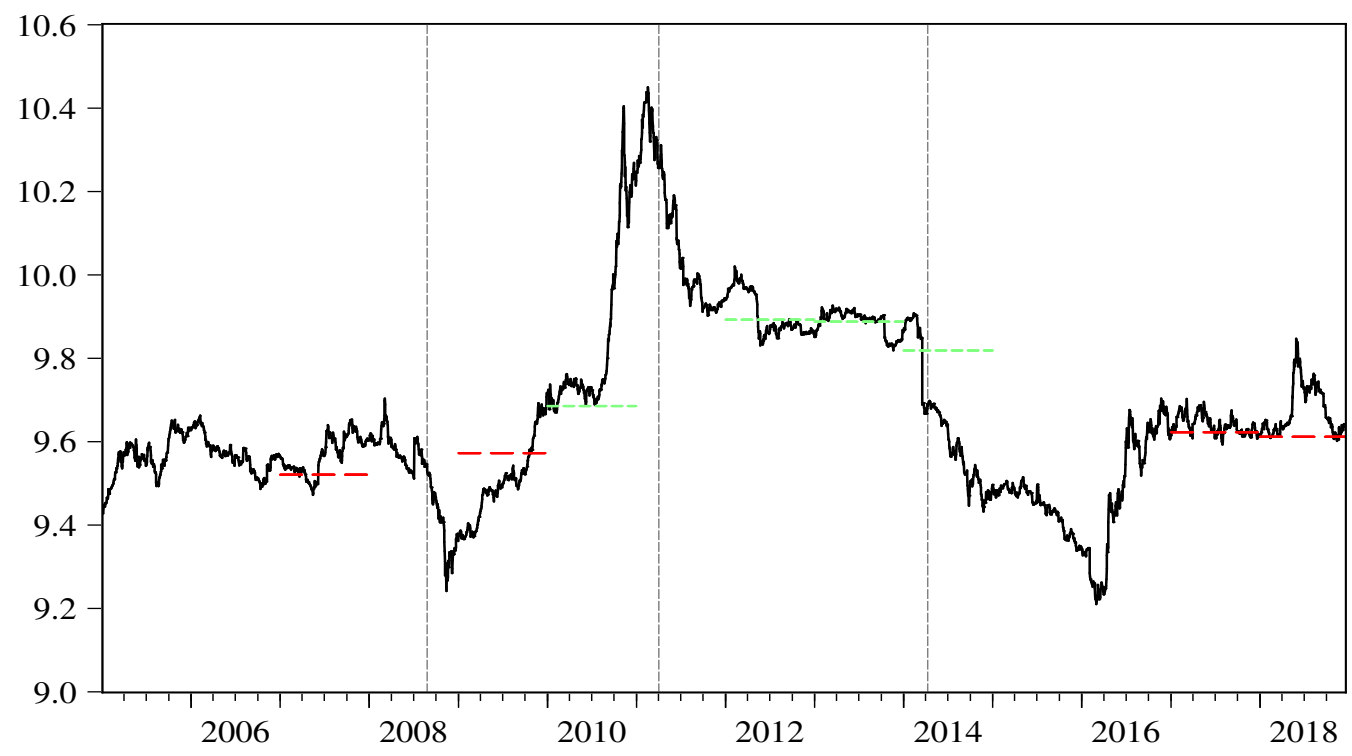

Cotton ---- Skew level $(\mathrm{p}<0.5)---$ Skew level $(\mathrm{p}>0.5)$

Figure 11: Skew phenomena in cotton futures market

Notes: This figure shows logarithmic prices of cotton futures (solid curve) with skew levels where skew probabilities are significantly larger than 0.5 (horizontal red dashed lines) and skew levels where skew probabilities are significantly smaller than 0.5 (horizontal green dashed lines). The vertical dashed lines illustrate policychange split points. The first one indicates that from August 21, 2008 to April 10, 2009, China purchased and stored 2.78 million tons of cotton. For the next two years, the NDRC suspended this policy to launch a cotton temporary state reserve program. The second one shows that the NDRC launched a cotton temporary state reserve program again on March 28, 2011. The last one indicates that the government for the first time proposed that the temporary reserve program for cotton would no longer be implemented beginning April 5, 2014 and changed the policy toward target price subsidies in the same year. The sample period extends from January 1, 2005 to December 22, 2018.

\section{Conclusion}

This study indicates that government intervention causes agricultural commodities markets show regulated and controlled characteristics. Traditional models have no way to characterize such features, while the skew OU model is good at depicting them. Our empirical results demonstrate that skew phenomena are quite significant and consistent with government intervention policy and other events that may influence price dynamics. For grains futures, the observed skew phenomena are most notable. For example, about 55\% of samples show skew phenomena. Grains are important to the national economy and people's livelihoods and therefore government intervention in this market is relatively high. We find that skew phenomena are relatively fewer for oilseed and soft futures, at only about $35 \%$ and $43 \%$, respectively. Prices of soybean and especially soymeal are affected more by international prices. Therefore, government intervention in these markets is less.

Development of asset pricing models for agricultural commodities is of importance to both a- 
Table 8: Parameter estimation results of skew OU model in white sugar futures market

\begin{tabular}{|c|c|c|c|c|c|c|c|c|c|c|}
\hline \multirow{2}{*}{ Year } & \multicolumn{7}{|c|}{ Skew OU estimates } & \multicolumn{3}{|c|}{ OU estimates } \\
\hline & $\hat{\kappa}$ & $\hat{\theta}$ & $\hat{\sigma}$ & $\hat{a}$ & $\hat{p}$ & $p$-value ${ }^{1}$ & $p$-value ${ }^{2}$ & $\hat{\kappa}_{0}$ & $\hat{\theta}_{0}$ & $\hat{\sigma}_{0}$ \\
\hline \multirow{3}{*}{2007} & & & & & & & & & 8.2132 & \\
\hline & & & & & & & & & & \\
\hline & {$[-0.3573]$} & {$[-0.0077]$} & {$[-1.2907]$} & {$[-1.0277]$} & {$[-1.1836]$} & {$[406.4146]$} & {$[404.7770]$} & & {$[1005.6696$} & \\
\hline \multirow{3}{*}{2008} & 8253 & 446 & 5 & 8.05 & 0.3962 & 0.0 & 260 & 2.0090 & 8.0269 & 0.16 \\
\hline & $(0.3273)$ & & & & & 5) & 120) & $.6527)$ & $(0.1117)$ & \\
\hline & {$[-0.2405]$} & {$[0.7285]$} & {$[0.1109]$} & {$[-0.6882]$} & {$[0.8514]$} & {$[307.0731]$} & [307.5168] & & [921.8421] & \\
\hline \multirow{3}{*}{2009} & & & & & & & 256 & 1.2272 & 8.6 & 0 \\
\hline & $(0.1139)$ & $(0$. & 1) & 9) & $(0.2584)$ & 892) & $(329.2522)$ & (1.0989) & $(0.2$ s & 73) \\
\hline & {$[-0.2483]$} & {$[-1.1$} & $39]$ & {$[1.3$} & {$[-0.24$} & {$[41:$} & $075]$ & & {$[965$.} & \\
\hline \multirow{3}{*}{2010} & & & & & & & & & & \\
\hline & 3) & $(0$ & & & $(0$ & $(44$ & $(45$ & $(14710)$ & $(0.1$ & $(0$ \\
\hline & & {$[-1$} & & & & & 074] & & & \\
\hline \multirow{3}{*}{2011} & & & & & & & & 1.0011 & & \\
\hline & $(0$. & & & & & & 426) & $2.7111)$ & & \\
\hline & {$[-0$} & {$[0$.} & 51] & {$[-0.3$} & {$[-0$.} & {$[40$} & $220]$ & & & \\
\hline \multirow{3}{*}{2012} & & & & & & & & & & \\
\hline & & & & & & & & & & \\
\hline & {$[1.2372]$} & {$[0.4510]$} & {$[-1.2258]$} & {$[0.7128]$} & {$[-0.6893]$} & {$[402.1081]$} & {$[396.8256]$} & & & \\
\hline \multirow{3}{*}{2013} & & & & & & & & 2.3296 & 8.48 & \\
\hline & 3) & & & & & $(35$ & 727) & $(2.1329)$ & & \\
\hline & & {$[1$.} & $01]$ & & {$[0$.} & {$[42$} & $322]$ & & {$[1072$} & \\
\hline \multirow{3}{*}{2014} & & & & & & & & & & \\
\hline & $(0.6168)$ & $.0040)$ & $(0.0072)$ & $(0.0050)$ & $(0.0430)$ & 549) & 7710) & $(26005)$ & $(0.032$ & \\
\hline & & & & & {$[-1.1062]$} & [421.1934] & $5632]$ & & {$[1008.0$} & \\
\hline \multirow{3}{*}{2015} & & & & & 0 & & & 492 & 8.6 & 0.1 \\
\hline & 47) & 19) & 1) & $(0$ & $(0$ & $(42$ & 688) & (0) -171$)$ & & \\
\hline & & & & & & & & & & \\
\hline \multirow{3}{*}{2016} & & & & & 402 & & 231 & & 8.7662 & 0.0985 \\
\hline & & & & & & & & $(1.1346)$ & & $(0.0046)$ \\
\hline & & & & & & {$[400.8676]$} & {$[396.9387]$} & & {$[1072.8612]$} & \\
\hline \multirow{3}{*}{2017} & 0.8412 & 8.7530 & 0.0761 & 8.7515 & 0.2512 & 0.0277 & 0.0939 & 1.1100 & 8.7074 & 0.0836 \\
\hline & $(0.26$ & & & & & $(455$ & $(406$ & $(2.0714)$ & & $(0.0039)$ \\
\hline & {$[-0.1809]$} & {$[-0.6550]$} & {$[-0.1576]$} & {$[-0.0784]$} & {$[0.1821]$} & [402.3210] & {$[402.9962]$} & & {$[1125$.} & \\
\hline \multirow{3}{*}{2018} & 1. & 8.5663 & 0.0815 & 8.4992 & 0.5021 & & & 1.4671 & 8.4371 & 0.08 \\
\hline & $(0$. & $(0$. & & $(0$. & & $(326$ & $275)$ & $(1.4010)$ & $(0.14$ & $(0.0039)$ \\
\hline & {$[0.3219]$} & {$[0.0090]$} & {$[1.3146]$} & {$[-0.0361]$} & {$[-0.1982]$} & [390.7068] & [401.1836] & & {$[1061.154$} & \\
\hline
\end{tabular}

Notes: Columns (2) to (6) report estimation results based on the skew OU model via Bayesian methods. Standard errors are reported in parentheses. CD statistics are reported in square brackets. Column (7) reports $p$-value ${ }^{1}$ for whether the estimator of the skew probability is significantly different from 0.5 . Column (8) reports $p$-value ${ }^{2}$ of the generalized quasi-likelihood ratio test for the null hypothesis that the OU model is more suitable than skew OU model. Corresponding test statistics are reported in the parenthesis. To save space, the critical values at the significance level of $10 \%$ used are reported in the square brackets. Columns (9) to (11) report estimation results based on OU model via MLE methods. The standard errors are reported in the parenthesis. Values of the maximized log-likelihood function are reported in the square brackets. The estimation period is based on daily observations from January 1, 2007 to December 22, 2018.

cademics and practitioners. Compared to the traditional OU model, the skew OU model captures specific regulatory characteristics, which is important for risk management in agricultural commodities markets. In addition, the model shows the effectiveness of government regulatory policy, as reflected by the magnitude of skew probabilities. Commodity futures have attracted more interest as a popular investment vehicle. This paper represents the first evaluation in the literature of the influence of 


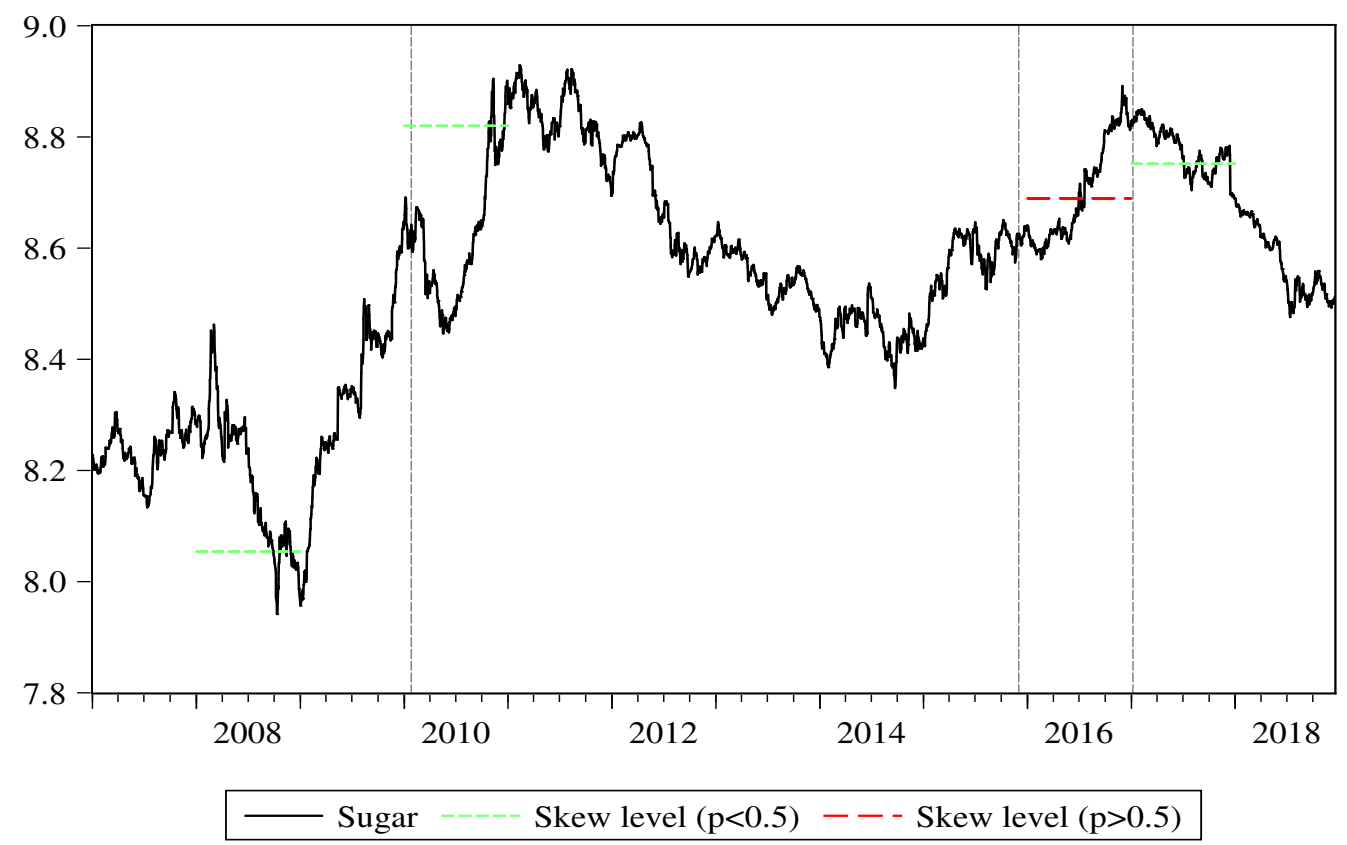

Figure 12: Skew phenomena in white sugar futures market

Note: This figure shows logarithmic prices of white sugar futures (solid curve) with the skew level, where skew probability is significantly larger than 0.5 (horizontal red dashed line) and skew levels where skew probabilities are significantly smaller than 0.5 (horizontal green dashed lines). The vertical dashed lines illustrate policyand yield-change split points. The first one indicates that from January 21, 2010, the government implemented large-scale destocking, putting prices under pressure. The second one shows that sugar yields fell more than expected in late 2015 and the yield and consumption gap significantly increased, forming strong support for sugar prices in the following year. The last one indicates that white sugar began a new production cycle in 2017 and the high probability of falling prices is not surprising. The sample period extends from January 1, 2007 to December 22, 2018.

government policies on the basis of skew level and skew probability.

We estimate skew level and skew probability using data from futures prices. It would be interesting to estimate these two parameters by combining information from both futures and corresponding options prices. This represents a promising path for future research. Implied information in futures options is often used as a forward-looking measure. Corn options, soymeal options, cotton options, and sugar options are traded in China. Thus, in the future, even more data will be available for research. Another interesting and important application of the skew OU model is to price agricultural commodity derivatives. We focus mainly on skew phenomena in the agricultural futures markets and hope to develop a more comprehensive model regarding the characteristics of regulation, seasonality, and stochastic volatility.

\section{Acknowledgements}

The authors thank the editor John W.Goodell and two anonymous referees for valuable comments and suggestions, which helped to improve the paper significantly. The authors are also indebted to participants in the seminar on Stochastic Processes and Financial Engineering at Nankai University 
for their valuable comments and discussions. Previous versions of this paper were presented at the 2019 Infinite Conference in the Asia-Pacific on Finance in Changing Global Environment, the 8th International Conference on Futures and Other Derivatives, 2019 SoFiE Summer School, and 2019 Financial Risk Management Annual Conference. We thank participants at the meetings for their valuable comments. Opinions expressed and any errors remain our own responsibility. This research was supported by the National Natural Science Foundation of China [grant numbers 71532001 and 11631004], and the Tianjin Social Science Planning Office, Key Project [grant number TJGL19-008]. 


\section{References}

Aggarwal, R., Lucey, B. M., 2007. Psychological barriers in gold prices? Rev. Financ. Econ. 16 (2), 217-230, https://doi.org/10.1016/j.rfe.2006.04.001.

Avery, C., Zemsky, P. B., 1998. Multidimensional uncertainty and herd behavior in financial markets. Am. Econ. Rev. 88 (4), 724-748, http://www.jstor.org/stable/117003.

Avriel, M., Hilscher, J., Raviv, A., 2013. Inflation derivatives under inflation target regimes. J. Futur. Mark. 33 (10), 911-938, https://doi.org/10.1002/fut.21568.

Bai, Y., Guo, Z., 2019. An empirical investigation to the "skew" phenomenon in stock index markets: Evidence from the Nikkei 225 and others. Sustain. 11 (24), 7219, https://doi.org/10.3390/su11247219.

Bardou, O., Martinez, M., 2010. Statistical estimation for reflected skew processes. Stat. Inference Stoch. Process. 13 (3), 231-248, https://doi.org/10.1007/s11203-010-9047-6.

Bessembinder, H., Coughenour, J. F., Seguin, P. J., Smoller, M. M., 1995. Mean reversion in equilibrium asset prices: Evidence from the futures term structure. J. Finance. 50 (1), 361-375, https://doi.org/10.1111/j.1540-6261.1995.tb05178.x.

Bo, L., 2013. First passage times of reflected Ornstein-Uhlenbeck processes with two-sided jumps. Queueing Syst. 73 (1), 105-118, https://doi.org/10.1007/s11134-012-9308-8.

Bo, L., Li, X., Wang, Y., Yang, X., 2013. On the conditional default probability in a regulated market with jump risk. Quant. Financ. 13 (12), 1967-1975, https://doi.org/10.1080/14697688.2013.815795.

Bo, L., Tang, D., Wang, Y., Yang, X., 2011a. On the conditional default probability in a regulated market: A structural approach. Quant. Financ. 11 (12), 1695-1702, https://doi.org/10.1080/14697680903473278.

Bo, L., Wang, Y., Yang, X., 2011b. Some integral functionals of reflected SDEs and their applications in finance. Quant. Financ. 11 (3), 343-348, https://doi.org/10.1080/14697681003785926.

Cai, N., Yang, X., 2018. International reserve management: A drift-switching reflected jump-diffusion model. Math. Financ. 28 (1), 409-446, https://doi.org/10.1111/mafi.12134.

Cai, N., Yang, X., 2020. A computational approach to first passage problems of reflected hyperexponential jump diffusion processes. INFORMS J. Comput. Forthcoming, https://doi.org/10.1287/ijoc.2020.0980.

Casassus, J., Collin-Dufresne, P., 2005. Stochastic convenience yield implied from commodity futures and interest rates. J. Finance. 60 (5), 2283-2331, https://doi.org/10.1111/j.1540-6261.2005.00799.x.

Chen, X., Chiang, T. C., 2020. Empirical investigation of changes in policy uncertainty on stock returns-Evidence from China's market. Res. Int. Bus. Financ. 53, 101183, https://doi.org/10.1016/j.ribaf.2020.101183.

Dowling, M. M., Cummins, M., Lucey, B. M., 2016. Psychological barriers in oil futures markets. Energy Econ. 53, 293-304, https://doi.org/10.1016/j.eneco.2014.03.022.

Fan, J., Jiang, J., Zhang, C., Zhou, Z., 2003. Time-dependent diffusion models for term structure dynamics. Stat. Sinca. 13 (4), 965-992, http://www3.stat.sinica.edu.tw/statistica/oldpdf/A13n44.pdf.

Farnsworth, H., Bass, R. F., 2003. The term structure with semi-credible targeting. J. Finance. 58 (2), 839-866, https://doi.org/10.1111/1540-6261.00548. 
Geman, H., 2005. Commodities and commodity derivatives: Modeling and pricing for agriculturals, metals and energy. John Wiley \& Sons Inc., New York.

Geweke, J., 1991. Evaluating the accuracy of sampling-based approaches to the calculation of posterior moments. Staff report, Federal Reserve Bank of Minneapolis.http://minneapolisfed.org/research/sr/sr148.pdf.

Han, Z., Hu, Y., Lee, C., 2016. Optimal pricing barriers in a regulated market using reflected diffusion processes. Quant. Financ. 16 (4), 639-647, https://doi.org/10.1080/14697688.2015.1034163.

Han, Z., Hu, Y., Lee, C., 2019. On pricing barrier control in a regime-switching regulated market. Quant. Financ. 19 (3), 491-499, https://doi.org/10.1080/14697688.2018.1480835.

Harrison, J. M., Shepp, L. A., 1981. On skew Brownian motion. Ann. Probab. 9 (2), 309-313, https://doi.org/10.1214/aop/1176994472.

Hirshleifer, D. A., 2001. Investor psychology and asset pricing. J. Finance. 56 (4), 1533-1597, https://doi.org/10.1111/0022-1082.00379.

Huo, R., Ahmed, A. D., 2018. Relationships between Chinese stock market and its index futures market: Evaluating the impact of QFII scheme. Res. Int. Bus. Financ. 44, 135-152, https://doi.org/10.1016/j.ribaf.2017.07.049.

Itô, K., Mckean, H. P., 1965. Diffusion processes and their sample paths. Springer, Berlin., https://doi.org/10.1007/978-3-642-62025-6.

Jian, Z., Deng, P., Luo, K., Zhu, Z., 2018. The effect of market quality on the causality between returns and volatilities: Evidence from CSI 300 index futures. J. Manag. Sci. Eng. 3 (1), 16-38, https://doi.org/10.3724/SP.J.1383.301002.

Klomp, J., 2020. The impact of Russian sanctions on the return of agricultural commodity futures in the EU. Res. Int. Bus. Financ. 51, 101073, https://doi.org/10.1016/j.ribaf.2019.101073.

Krugman, P., 1991. Target zones and exchange rate dynamics. Q. J. Econ. 106 (3), 669-682, https://doi.org/10.2307/2937922.

Lee, C., Song, J., 2016. On drift parameter estimation for reflected fractional Ornstein-Uhlenbeck processes. Stoch. 88 (5), 751-778, https://doi.org/10.1080/17442508.2016.1143472.

Lejay, A., Pichot, G., 2012. Simulating diffusion processes in discontinuous media: A numerical scheme with constant time steps. J. Comput. Phys. 231 (21), 7299-7314, https://doi.org/10.1016/j.jcp.2012.07.011.

Li, L., Linetsky, V., 2014. Time-changed Ornstein-Uhlenbeck processes and their applications in commodity derivative models. Math. Financ. 24 (2), 289-330, https://doi.org/10.1111/mafi.12003.

Liang, T., Chai, J., Zhang, Y., Zhang, Z. G., 2019. Refined analysis and prediction of natural gas consumption in China. J. Manage. Sci. Eng. 4 (2), 91-104, https://doi.org/10.1016/j.jmse.2019.07.001.

MacDonald, S., Gale, F., Hansen, J., 2015. Cotton policy in China. MPRA paper, University Library of Munich, Germany. 70863, 1-37, https://mpra.ub.uni-muenchen.de/70863/.

Mohanty, S. K., Mishra, S., 2020. Regulatory reform and market efficiency: The case of Indian agricultural commodity futures markets. Res. Int. Bus. Financ. 52, 101145, https://doi.org/10.1016/j.ribaf.2019.101145.

Nakatsuma, T., 2000. Bayesian analysis of ARMA-GARCH models: A Markov chain sampling approach. J. Econom. 95 (1), 57-69, https://doi.org/10.1016/S0304-4076(99)00029-9. 
Narayan, P. K., Narayan, S., Popp, S., 2011. Investigating price clustering in the oil futures market. Appl. Energy 88 (1), 397-402, https://doi.org/10.1016/j.apenergy.2010.07.034.

Perera, D., Bialkowski, J., Bohl, M. T., 2020. Does the tea market require a futures contract? Evidence from the Sri Lankan tea market. Res. Int. Bus. Financ. 54, 101290, https://doi.org/10.1016/j.ribaf.2020.101290.

Protter, P., 2004. Stochastic integration and differential equation. Springer, Berlin, Heidelberg, https://doi.org/10.1007/978-3-662-02619-9.

Revuz, D., Yor, M., 1999. Continuous martingales and Brownian motion. 3rd ed. Springer, Berlin, https://doi.org/10.1007/978-3-662-06400-9.

Ritter, C., Tanner, M. A., 1992. Facilitating the Gibbs sampler: The Gibbs stopper and the Griddy-Gibbs sampler. J. Am. Stat. Assoc. 87 (419), 861-868, https://doi.org/10.1080/01621459.1992.10475289.

Schwartz, E. S., 1997. The stochastic behavior of commodity prices: Implications for valuation and hedging. J. Finance. 52 (3), 923-973, https://doi.org/10.1111/j.1540-6261.1997.tb02721.x.

Schwartz, E. S., Smith, J. E., 2000. Short-term variations and long-term dynamics in commodity prices. Manage. Sci. 46 (7), 893-911, https://doi.org/10.1287/mnsc.46.7.893.12034.

Svensson, L. E., 1991. The simplest test of target zone credibility. IMF Econ Rev. 38, 655-665, https://doi.org/10.2307/3867162.

Wang, S., Song, S., Wang, Y., 2015. Skew Ornstein-Uhlenbeck processes and their financial applications. J. Comput. Appl. Math. 273 (1), 363-382, https://doi.org/10.1016/j.cam.2014.06.023.

Westerhoff, F., 2003. Anchoring and psychological barriers in foreign exchange markets. J. Behav. Financ. 4 (2), 65-70, https://doi.org/10.1207/S15427579JPFM0402_03.

Yang, X., Ren, G., Wang, Y., Bo, L., Li, D., 2016. Modeling the exchange rates in a target zone by reflected Ornstein-Uhlenbeck process. Available at SSRN 2107686,https://doi.org/10.2139/ssrn.2107686.

Zhuo, X., 2018. Pricing research and empirical test based on the skew-extended stochastic interest rate models. Unpublished doctor thesis, Nankai University.

Zhuo, X., Menoukeupamen, O., 2017. Efficient piecewise trees for the generalized skew Vasicek model with discontinuous drift. Int. J. Theor. Appl. Financ. 20 (4), 1750028, https://doi.org/10.1142/S0219024917500285.

\section{Appendices}

\section{A Definition of the Symmetric Local Time}

Here we recall the definition of the symmetric local time from Protter (2004). Let sign $(x)$ be the sign function defined by:

$$
\operatorname{sign}(x)= \begin{cases}1, & \text { if } 0<x, \\ -1, & \text { if } x \leq 0\end{cases}
$$


Then the right local time of $X$ at the level $a$ is:

$$
L_{t}^{X}(a) \triangleq\left|X_{t}-a\right|-\left|X_{0}-a\right|-\int_{0}^{t} \operatorname{sign}\left(X_{s}-a\right) d X_{s}
$$

with the left local time $L_{t}^{X}(a-) \triangleq \lim _{b \uparrow a} L_{t}^{X}(b)$.

Hence, the symmetric local time $\hat{L}_{t}^{X}(a)$ is given by:

$$
\hat{L}_{t}^{X}(a) \triangleq\left[L_{t}^{X}(a)+L_{t}^{X}(a-)\right] / 2 .
$$

\section{B Transformation of the Skew OU Process}

Since the function $G(\cdot)$ is the difference of two convex functions, we apply the generalized Itô formula (see Revuz and Yor, 1999) to process $Y_{t}$, and obtain:

$$
Y_{t}=G\left(X_{0}\right)+\frac{1}{2} \int_{0}^{t}\left(G^{\prime}\left(X_{s}+\right)+G^{\prime}\left(X_{s}-\right)\right) d X_{s}+\frac{1}{2} \int_{\mathbf{R}} \hat{L}_{t}^{X}(z) \mu(d z)
$$

where $G^{\prime}(x+)\left(G^{\prime}(x-)\right)$ is the right (left) derivative of $G(x), \mu$ is the signed measure (when restricted to compacts), which is the second derivative of $G$ in the generalized function sense with the properties $\mu((a, b])=G^{\prime}(b+)-G^{\prime}(a-)$ and $\mu(\{x\})=G^{\prime}(x+)-G^{\prime}(x-)$. Further, if $G(\cdot)$ is the bounded Borel measurable function, we have the occupation time formula:

$$
\int_{\mathbf{R}} \hat{L}_{t}^{X}(z) \mu(d z)=\int_{0}^{t} G^{\prime \prime}\left(X_{s}\right) d\langle X\rangle_{s},
$$

then we have:

$$
\begin{aligned}
Y_{t}= & G\left(X_{0}\right)+\frac{1}{2} \int_{0}^{t}\left(G^{\prime}\left(X_{s}+\right)+G^{\prime}\left(X_{s}-\right)\right) 1_{\left\{X_{s} \neq a\right\}} d X_{s}+\frac{1}{2} \int_{\mathbf{R} \backslash\{a\}} \hat{L}_{t}^{X}(z) \mu(d z) \\
& +\frac{1}{2} \int_{0}^{t}\left(G^{\prime}\left(X_{s}+\right)+G^{\prime}\left(X_{s}-\right)\right) 1_{\left\{X_{s}=a\right\}} d X_{s}+\frac{1}{2} \int_{\{a\}} \hat{L}_{t}^{X}(z) \mu(d z) \\
= & G\left(X_{0}\right)+\int_{0}^{t} G^{\prime}\left(X_{s}\right)\left(\kappa\left(\theta-X_{s}\right) d s+\sigma d W_{s}\right)+\frac{1}{2} \int_{0}^{t} G^{\prime \prime}\left(X_{s}\right) d\langle X\rangle_{s} \\
& +\frac{1}{2}\left(G^{\prime}(a+)+G^{\prime}(a-)\right)(2 p-1) \hat{L}_{t}^{X}(a)+\frac{1}{2}\left(G^{\prime}(a+)-G^{\prime}(a-)\right) \hat{L}_{t}^{X}(a) \\
= & G\left(X_{0}\right)+\int_{0}^{t} G^{\prime}\left(X_{s}\right)\left(\kappa\left(\theta-X_{s}\right) d s+\sigma d W_{s}\right)+\frac{1}{2} \int_{0}^{t} G^{\prime \prime}\left(X_{s}\right) \sigma^{2} d s .
\end{aligned}
$$

We finally get the new process $Y_{t}$ without the local time component.

Remark B.1 The last equality holds because we use the fact that the skew diffusion admits the fol- 
lowing boundary (see, for example, Harrison and Shepp, 1981):

$$
p G^{\prime}(a+)-(1-p) G^{\prime}(a-)=0 .
$$

We rewrite the right-hand side of Eq.(B.1) in terms of $Y_{t}$, then the skew OU process $X_{t}$ defined in Eq.(2.3) is transformed to a tractable piecewise process $Y_{t}$ satisfying the following SDE:

$$
d Y_{t}= \begin{cases}\kappa\left[(1-p) \theta+p a-Y_{t}\right] d t+(1-p) \sigma d W_{t}, & \text { if } a \leq Y_{t}, \\ \kappa\left[p \theta+(1-p) a-Y_{t}\right] d t+p \sigma d W_{t}, & \text { if } Y_{t}<a .\end{cases}
$$

\section{Bayesian Estimation of the Skew OU Process}

\section{C.1 Conditional Distribution of Instantaneous Return}

Conditional on $\theta, \sigma, p$ and $a$, the conditional posterior distribution of mean-reversion speed $\kappa$ should be as follows:

$$
\kappa \mid X, \theta, \sigma^{2}, p, a \sim \mathcal{N}\left(\widehat{\mu}_{\kappa}, \widehat{\sigma}_{\kappa}^{2}\right),
$$

where

$$
\begin{aligned}
\widehat{\mu}_{\kappa}= & \left\{\frac{p^{2} \sum_{i \in N_{1}} y_{t_{i}}\left\{\left[-x_{t_{i}}+((1-p) \theta+p a)\right] \triangle t\right\}}{p^{2}(1-p)^{2} \sigma^{2} \triangle t}\right. \\
& \left.+\frac{(1-p)^{2} \sum_{i \in N_{2}} y_{t_{i}}\left\{\left[-x_{t_{i}}+(p \theta+(1-p) a)\right] \triangle t\right\}}{p^{2}(1-p)^{2} \sigma^{2} \triangle t}+\frac{\mu_{\kappa}}{\sigma_{\kappa}^{2}}\right\} \widehat{\sigma}_{\kappa}^{2}, \\
\widehat{\sigma}_{\kappa}^{-2}= & \frac{p^{2} \sum_{i \in N_{1}}\left\{\left[x_{t_{i}}-((1-p) \theta+p a)\right] \triangle t\right\}^{2}}{p^{2}(1-p)^{2} \sigma^{2} \triangle t} \\
& +\frac{(1-p)^{2} \sum_{i \in N_{2}}\left\{\left[x_{t_{i}}-(p \theta+(1-p) a)\right] \triangle t\right\}^{2}}{p^{2}(1-p)^{2} \sigma^{2} \triangle t}+\frac{1}{\sigma_{\kappa}^{2}} .
\end{aligned}
$$

Similarly, given $\kappa, \sigma, p$ and $a$, the conditional posterior distribution of long-term mean $\theta$ is:

$$
\theta \mid X, \kappa, \sigma^{2}, p, a \sim \mathcal{N}\left(\widehat{\mu}_{\theta}, \widehat{\sigma}_{\theta}^{2}\right)
$$

where

$$
\begin{aligned}
\widehat{\mu}_{\theta}= & \left\{\frac{p^{2} \sum_{i \in N_{1}}\left[\kappa(1-p) \triangle t\left(y_{t_{i}}+\kappa \triangle t x_{t_{i}}-\kappa p a \triangle t\right)\right]}{p^{2}(1-p)^{2} \sigma^{2} \triangle t}\right. \\
& \left.+\frac{(1-p)^{2} \sum_{i \in N_{2}}\left\{\kappa p \triangle t\left[y_{t_{i}}+\kappa \triangle t x_{t_{i}}-\kappa(1-p) a \triangle t\right]\right\}}{p^{2}(1-p)^{2} \sigma^{2} \triangle t}+\frac{\mu_{\theta}}{\sigma_{\theta}^{2}}\right\} \widehat{\sigma}_{\theta}^{2}, \\
\widehat{\sigma}_{\theta}^{-2}= & \frac{\kappa^{2} \triangle t N}{\sigma^{2}}+\frac{1}{\sigma_{\theta}^{2}} .
\end{aligned}
$$




\section{C.2 Conditional Distribution of Volatility}

Conditional on $\kappa, \theta, p$ and $a$, the posterior distribution of $\sigma^{2}$ is:

$$
\sigma^{2} \mid X, \kappa, \theta, p, a \sim \mathcal{I} \mathcal{G}\left(\widehat{\alpha}_{\sigma}, \widehat{\lambda}_{\sigma}\right)
$$

where

$$
\begin{aligned}
\widehat{\alpha}_{\sigma}= & \frac{n_{1}}{2}+\frac{n_{2}}{2}+\alpha_{\sigma}, \\
\widehat{\lambda}_{\sigma}= & \frac{1}{2(1-p)^{2} \triangle t} \sum_{i \in N_{1}}\left\{y_{t_{i}}+\kappa \Delta t x_{t_{i}}-\kappa[(1-p) \theta+p a] \triangle t\right\}^{2} \\
& +\frac{1}{2 p^{2} \triangle t} \sum_{i \in N_{2}}\left\{y_{t_{i}}+\kappa \triangle t x_{t_{i}}-\kappa[p \theta+(1-p) a] \triangle t\right\}^{2}+\lambda_{\sigma} .
\end{aligned}
$$

\section{C.3 Conditional Distribution of Skew Level}

Because it is hard to find the conjugate prior for the skew level $a$, the Griddy-Gibbs sampler is chosen here in accordance with Ritter and Tanner (1992). The conditional posterior distribution of $a$ is a distribution with probability function:

$$
p\left(a_{i} \mid X, \kappa, \theta, \sigma^{2}, p\right)=\frac{L\left(X \mid \kappa, \theta, \sigma, a_{i}, p\right)}{\sum_{j=1}^{n} L\left(X \mid \kappa, \theta, \sigma, a_{j}, p\right)}
$$

\section{C.4 Conditional Distribution of the Skew Probability}

Analogously, conditional on $\kappa, \theta, \sigma$ and $a$, we calculate the density of skew probability $p$ as follows:

$$
p\left(p_{i} \mid X, \kappa, \theta, \sigma^{2}, a\right)=\frac{L\left(X \mid \kappa, \theta, \sigma, a, p_{i}\right)}{\sum_{j=1}^{n} L\left(X \mid \kappa, \theta, \sigma, a, p_{j}\right)}
$$

\section{Convergence Diagnostic Test}

We care that if the Markov chain of parameters generated from the Gibbs sampler converges to the real posterior density function $f(\Theta \mid X)$.

As Geweke (1991) and Nakatsuma (2000) worked out, after discarding the first $d$ runs for a sequence, we can test the convergence through comparing the first $m_{1}$ values in the remainder with the last $m_{2}$ ones. Formally, the CD statistics are defined as:

$$
C D=\left(m_{1}^{-1} \sum_{i=1+d}^{m_{1}+d} G(i)-m_{2}^{-1} \sum_{i=m+d-m_{2}+1}^{m+d} G(i)\right) /\left[\widehat{S}_{G}^{1}(0) / m_{1}+\widehat{S}_{G}^{2}(0) / m_{2}\right]^{\frac{1}{2}}
$$

where $\widehat{S}_{G}^{i}(\cdot)$ is the spectrum density estimate for $m_{i}$ runs. Let $\left(m_{1}+m_{2}\right) / m<1$ and fix the ratios $m_{1} / m$ and $m_{2} / m$. If the sequence $\{G(i), i=d+1, \cdots, d+m\}$ is stationary, then applying the central 
limit theorem, we have

$$
C D \rightarrow \mathcal{N}(0,1) \text { when } m \rightarrow \infty
$$

We set the ratios $m_{1} / m$ and $m_{2} / m$ to be 0.1 and 0.5 , as in Geweke (1991). 